\title{
La géovisualisation de données massives sur le Web : entre avancées technologiques et évolutions cartographiques
}

The geovisualisation of big data on the Web: technological advances and cartographic evolutions

La geovisualización de macrodatos (o big data) en la Web : entre los avances tecnológicos y la evolución cartográfica

\section{Boris Mericskay}

\section{(2) OpenEdition}

\section{Journals}

Édition électronique

URL : https://journals.openedition.org/mappemonde/5595

DOI : $10.4000 /$ mappemonde.5595

ISSN : 1769-7298

\section{Éditeur}

UMR ESPACE

Référence électronique

Boris Mericskay, «La géovisualisation de données massives sur le Web : entre avancées technologiques et évolutions cartographiques », Mappemonde [En ligne], 131 | 2021, mis en ligne le 08 juillet 2021, consulté le 14 juillet 2021. URL : http://journals.openedition.org/mappemonde/5595 ; DOI : https://doi.org/10.4000/mappemonde.5595

Ce document a été généré automatiquement le 14 juillet 2021. 


\section{La géovisualisation de données massives sur le Web : entre avancées technologiques et évolutions cartographiques}

The geovisualisation of big data on the Web: technological advances and cartographic evolutions

La geovisualización de macrodatos (o big data) en la Web : entre los avances tecnológicos y la evolución cartográfica

Boris Mericskay

\section{Introduction}

1 Avec le développement exponentiel du Web et l'émergence des données massives, la cartographie a profondément évolué tant d'un point de vue des techniques, des usages, qu'au niveau des modes de représentation (Crampton et al., 2013; Veenendaal et al., 2017). Sur le géoweb, la carte ne se positionne plus uniquement comme un outil de représentation de données géographiques, mais davantage comme une interface pour naviguer et explorer des contenus numériques toujours plus nombreux et diversifiés (Joliveau et al. 2013 ; Mericskay, 2016).

2 Afin de répondre aux défis de géovisualisation de données massives (volume, lisibilité, rendu graphique, interaction, etc.), les solutions de cartographie en ligne tendent peu à peu à s'éloigner du monde de la cartographie et des SIG pour tendre vers celui de l'exploration et de la visualisation de données (Elwood, 2011). D'un côté, les données spatiales sont au centre d'importants enjeux pour les géants du numérique qui développent de nouvelles approches et de nouveaux outils pour leur manipulation et leur analyse. De l'autre, les profils des cartographes du Web se diversifient, à l'image 
des développeurs Web, des data scientists ou des data journalists pour qui la carte est un moyen, parmi d'autres, pour représenter de l'information.

3 Malgré un discours dominant axé sur le renouvellement et l'innovation autour des méthodes et des outils, le développement de cette cartographie en ligne "guidée par les données»(Data-Driven Maps), ne marque pas véritablement de ruptures dans la manière de représenter des données via des cartes. La nouvelle génération d'outils emprunte pour beaucoup à la fois aux langages et aux systèmes de signes cartographiques plus «classiques » à l'image de la sémiologie graphique (Bertin, 1967, Lambert et Zanin, 2016), comme aux méthodes d'agrégation issues de l'analyse spatiale de la géovisualisation ou de la visualisation d'informations. La principale évolution se situe davantage au niveau des performances des outils qui autorisent le traitement et l'affichage de gros volumes de données géographiques au sein des environnements en ligne.

4 Si la question de l'innovation cartographique sur le Web est étroitement liée aux avancées technologiques, celles des "nouveaux cartographes » et des nouveaux statuts des cartes sur le Web (Elwood et Leszczynski, 2013) doivent être prises en compte et mises en perspective au regard des usages actuels des cartes en ligne. Et au-delà de l'instrumentation, les modes de représentation cartographique présents sur le géoweb doivent être questionnés tant au niveau des méthodes de conception, des formes d'interactions qu'ils proposent que de leur efficience.

Cet article propose en ce sens une réflexion sur les nouvelles modalités de conception et d'utilisation des cartes en ligne à l'heure des données volumineuses. Par un tour d'horizon des tendances et des enjeux de la cartographie en ligne, l'objectif est de questionner les évolutions techniques en cours et les changements qu'elles produisent sur les logiques et pratiques de représentations cartographiques sur le Web, notamment pour les big data.

6 Trois principales évolutions sont ici présentées et discutées. Les tuiles vectorielles qui transforment les modalités d'affichage, de personnalisation et de manipulation des données spatiales sur le Web sont préalablement présentées et mises en perspectives avec les usages émergents. Les modes de représentation basés sur l'agrégation spatiale comme forme de réponse aux enjeux de visualisation de volumes toujours plus grands de données spatiales sont ensuite abordés et discutés (clusters, carte de chaleur, carroyage). Enfin, la dernière partie s'intéresse plus spécifiquement au réinvestissement du champ de la représentation en trois dimensions de données spatiales permis notamment par le développement du WebGL (bâtiments, données statistiques, flux).

\section{Les tuiles vectorielles : de l'innovation technique à l'innovation cartographique}

\section{Retour sur le principe des tuiles vectorielles}

7 Avec la multiplication de données spatiales, toujours plus précises et hétérogènes, l'un des principaux enjeux de la cartographie en ligne se trouve dans la mise en place de solutions performantes permettant à la fois un transfert rapide des données, un rendu graphique fluide et des formes d'interaction poussées. L'une des voies les plus 
explorées pour adapter la cartographie en ligne aux enjeux des données massives repose dans la mobilisation des tuiles vectorielles (Yao et Li, 2018 ; Zouhar et Senner, 2019 ; Breunig et al., 2020). Cette technique émergente pour la diffusion de données spatiales en ligne vient profondément modifier les modalités de conception, de publication et d'utilisation des cartes sur le Web (Antoniou et al.. 2009 ; Gaffuri, 2012) ; Li et al., 2017).

D'un point de vue technique, les tuiles vectorielles, comme les tuiles raster, sont basées sur le principe d'une mosaïque, où le monde est découpé en tuiles dont le contenu s'adapte au niveau d'échelle de visualisation. Mais à la différence d'une tuile raster, qui repose sur la transmission d'images, le tuilage vectoriel permet le transfert d'objets géographiques (points, lignes, polygones), découpés en tuiles et généralisés en fonction du niveau d'échelle, auxquels sont associés des attributs (figure 1). On passe ainsi d'une méthode de diffusion de fonds de carte dont la mise en forme n'est pas modifiable à une logique de mise à disposition de données spatiales manipulables et personnalisables (Beukelaar, 2018 ; Zouhar et Senner, 2019).

Figure 1. Principe des tuiles vectorielles

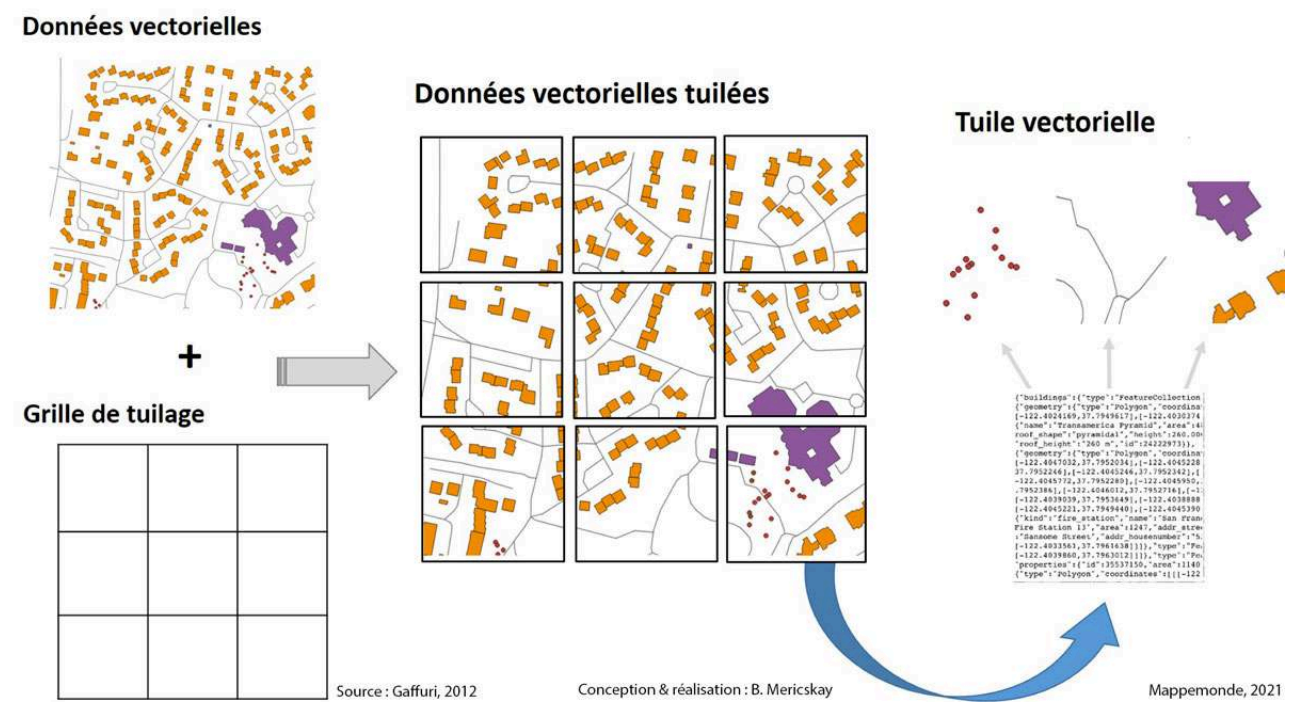

Boris Mericskay, inspiré de Gaffuri, 2012

9 Souvent précurseur dans le domaine du Webmapping, Google a introduit dès 2010 le tuilage vectoriel au sein du service Google Maps, tant la masse d'informations à afficher sur la carte était grande. Depuis, la plupart des grands acteurs du géospatial se sont lancés dans le tuilage vectoriel dans le but d'améliorer, de fluidifier et de renouveler leurs services cartographiques (Microsoft, ESRI, Mapbox, HERE...). Côté institutionnel, des initiatives mobilisant cette technologie commencent à émerger comme avec le nouveau géoportail suisse ${ }^{1}$, le service Open Zoomstack ${ }^{2}$ de l'Ordnance Survey, les services de tuiles vectorielles d'Etalab ${ }^{3}$ ou l'IGN qui a récemment lancé un géoservice de flux vecteur tuilé4. 
Figure 2. (a) géoportail suisse en tuiles raster ; (b) géoportail suisse en tuiles vecteur
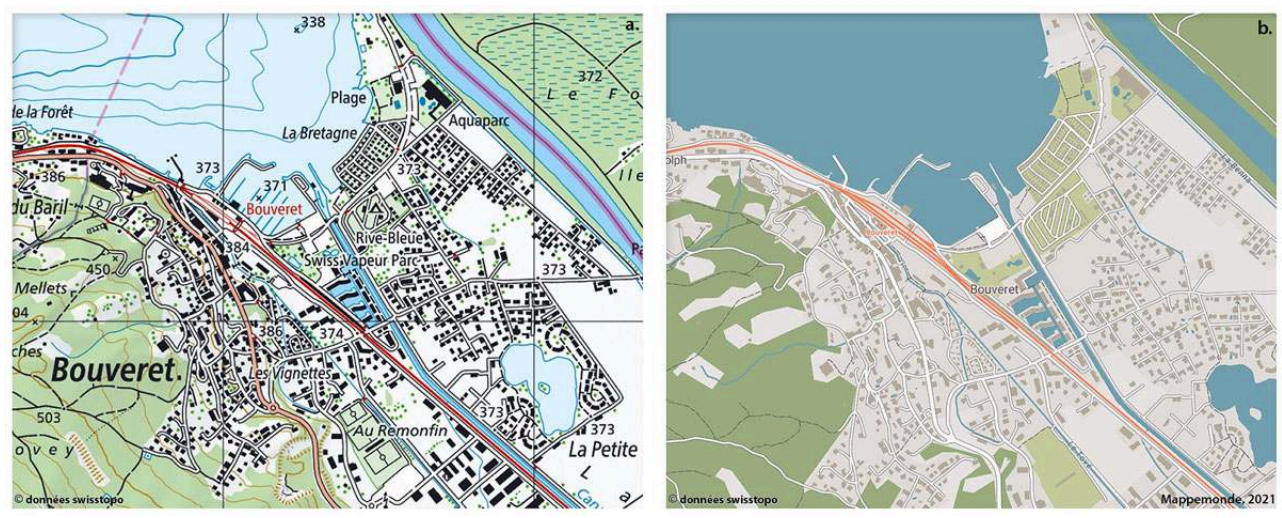

(a) https://map.geo.admin.ch/?lang=fr\&topic=ech\&bgLayer=ch.swisstopo.pixelkartefarbe\&layers=ch.swisstopo.zeitreihen,ch.bfs.gebaeude_wohnungs_register,ch.bav.haltestellenoev,ch.swisstopo.swisstlm3d-wanderwege\&layers_opacity=1,1,1,0.8\&layers_visibility=false, ; (b) https://test.map.geo.admin.ch/?

topic=ech\&lang=fr\&layers=ch.swisstopo.zeitreihen,ch.bfs.gebaeude_wohnungs_register,ch.bav.haltestellenoev,ch.swisstopo.swisstlm3d-

wanderwege\&layers_opacity=1,1,1,0.8\&layers_visibility=false,false,false,false\&layers_timestam

10 L'utilisation des tuiles vectorielles par rapport aux tuiles raster présente de nombreux avantages tant au niveau des performances, des capacités de rendu que de l'interactivité (Lienert et al., 2012). En premier lieu, les tuiles vectorielles, de manière similaire aux tuiles raster, reposent sur une logique pyramidale où les objets géographiques affichés et la précision des tracés cartographiques s'adaptent en fonction du niveau de zoom. Les géométries des lignes et des polygones peuvent ainsi être simplifiées ${ }^{5}$ et filtrées au sein de tuiles (pré-calculées) selon l'échelle de visualisation afin d'optimiser la vitesse de transmission (Taraldsvik, 2012). Le tuilage vectoriel assure également une plus grande rapidité d'exécution, car seules les données nécessaires à l'affichage sont transmises. Enfin, les fichiers de tuiles vectorielles sont bien plus légers que les tuiles raster dans la mesure où il s'agit de format texte et non d'images.

11 Cette réduction importante du volume et de la taille des données téléchargées vient réduire les temps de transfert, optimisant ainsi l'expérience de navigation des utilisateurs au sein des applications cartographiques en ligne (Shang, 2015; Farkas, 2019). En complément, l'accélération matérielle pour le rendu graphique, qui s'effectue côté client, offre des capacités d'affichage décuplées. Il est ainsi plus simple de publier des données aux géométries complexes (à l'image des polygones) qui peuvent contenir des millions d'entités. Avec les tuiles vectorielles, la géovisualisation et l'exploration de contenus géographiques volumineux et diversifiés se font plus fluides et plus rapides tout en permettant de conserver une bonne qualité graphique et un niveau de précision avancé, quel que soit le niveau d'échelle (Netek et al., 2020).

\section{De nouvelles formes d'interaction entre usagers et données géographiques volumineuses}

12 Au-delà du volume et de la rapidité, les cartes en ligne se font également plus interactives dans la mesure où les objets géographiques transmis via les tuiles sont manipulables et interrogeables. Cette différence, fondamentale par rapport aux tuiles 
raster, offre de nombreuses possibilités d'interaction entre l'usager et les données affichées (filtre, recherche, sélections attributaires ou spatiales, affichage des propriétés de l'objet). Sur le géoweb, les usagers ne lisent pas seulement les cartes, ils les utilisent, les modifient, les interrogent et les personnalisent en fonction de leurs objectifs (Muehlenhaus, 2013).

Plusieurs exemples de cartes en ligne basées sur les tuiles vectorielles peuvent être présentés afin d'illustrer les usages de cette technique pour la navigation et l'exploration de jeux de données géographiques volumineux. La carte de France Pixel ${ }^{6}$ permet par exemple d'explorer les données carroyées de l'INSEE (plus de 2 millions de carreaux) de manière dynamique et interactive. L'usager peut choisir les variables à cartographier (population, revenus, classe d'âge, etc.), visualiser ces données à différentes échelles d'agrégation et obtenir à la volée une synthèse des indicateurs de chaque carreau, grâce à des tuiles pré-calculées pour différentes échelles d'affichage. Dans la même logique, la carte en ligne France Pixel Bâti (https://www.comeetie.fr/ galerie/francepixelsbati/\#5.7/47/2.3) offre la possibilité de visualiser à différents niveaux d'agrégation les caractéristiques de 45 millions de bâtiments (âge, type de toiture, usage, etc.) issus de la BDTOPO de l'IGN. La carte proposée sur le site OneSoilMap ${ }^{7}$ permet de son côté de visualiser et d'interroger plus de 60 millions de parcelles agricoles à travers le monde, qui sont générées et enrichies sémantiquement par intelligence artificielle à partir de données satellitaires. La carte Colouring.London ${ }^{8}$ offre quant à elle la possibilité d'explorer les 3,5 millions de bâtiments de Londres par la génération de cartes thématiques à la volée selon différentes caractéristiques (âge, usage, taille, performances énergétiques...).

Certains jeux de données publics relatifs à l'urbanisme et à la volumétrie importante commencent à être proposés sous la forme de tuiles vectorielles. La plateforme geo.data.gouv, rattachée à Etalab, a par exemple développé une carte dynamique et interactive qui permet de visualiser de manière fluide et d'interroger plus 90 millions de sections et parcelles cadastrales 9 . Dans la même logique, Etalab propose depuis 2019 une carte interactive des demandes de valeurs foncières qui offre la possibilité d'explorer et d'interroger plus de 15 millions de transactions foncières opérées entre 2014 et 2019 sur le territoire français ${ }^{10}$.

Dernier exemple caractéristique des évolutions en cours, la centralisation sein d'un service de tuiles vectorielles de 4 millions de périmètres administratifs, postaux, électoraux et statistiques, à différentes échelles et couvrant le monde entier ${ }^{11}$. L'objectif est ici de fournir un référentiel vectoriel harmonisé et à jour de découpages territoriaux pour pouvoir produire rapidement des cartes d'analyse et mettre en place des applications de géovisualisation. Ce service d'un nouveau genre, payant en fonction des usages, représente une réelle avancée dans la mise à disposition de contours hétérogènes, à la base de production de nombreuses géovisualisations, qui sont ici directement mobilisables en ligne pour le traitement cartographique d'élections ${ }^{12}$ ou de données produites par les agences de statistiques publiques.

\section{La personnalisation des fonds de carte comme nouvelle pratique}

16 L'autre atout majeur des tuiles vectorielles réside dans la possibilité de modifier à la demande la symbologie des entités spatiales grâce aux feuilles de styles (Hayat, 2017). Il est ainsi possible de construire de manière dynamique ses propres fonds de carte selon 
ses goûts, la thématique traitée ou une charte graphique sans avoir besoin de régénérer des tuiles. Ce point est particulièrement intéressant dans la mesure où la conception des fonds de cartes, comme éléments constitutifs des cartes en ligne, est toujours plus centrée autour des utilisateurs (Schmidt et Weiser, 2012 ; Beukelaar, 2018).

Cette manière de concevoir des fonds de carte renvoie également à une logique de géovisualisation multiscalaire où les éléments présents comme la symbologie s'adaptent à l'échelle de visualisation. Déjà présente dans le cadre des tuiles raster, cette approche est largement réinvestie avec les tuiles vectorielles (Dubrava, 2017). Cette nouvelle dynamique s'appuie pour beaucoup sur les données libres d'OpenStreetMap à l'image des fonds de carte produits au début des années 2010 par des entreprises comme Stamen ${ }^{13}$ ou Mapzen ${ }^{14}$ qui ont lancé une nouvelle manière d'envisager et d'utiliser les fonds de carte en introduisant une dimension stylistique.

Des styles minimalistes aux styles vintage ou monochromes, des milliers de fonds de carte déjà mis en forme sont actuellement accessibles via des plateformes comme Snazzy Maps ${ }^{15}$, Mapbox Gallery ${ }^{16}$ ou Open Map Styles ${ }^{17}$. En plus de styles déjà configurés, il est aussi possible d'éditer ses propres styles de fond de carte en modifiant les données présentes, leur symbologie ou les étiquettes en fonction des niveaux de zoom. La boîte à outils en ligne pour personnaliser facilement et rapidement dans des environnements interfacés est assez riche à l'image de Mapbox Studio ${ }^{18}$ (figure 3), Jawg Editor $^{19}$, Vector Tile style Editor ${ }^{20}$ de ESRI, Map Style ${ }^{21}$ de Google, Maputnik ${ }^{22}$ ou Map Style Sheet Editor ${ }^{23}$ de Microsoft. Une fois conçus, ces fonds de carte personnalisés et stylisés sont mobilisables en ligne ou au sein de logiciels SIG ouvrant ainsi la voie à des usages plus larges.

Figure 3. Interface du service en ligne de personnalisation de fonds de carte Mapbox Studio

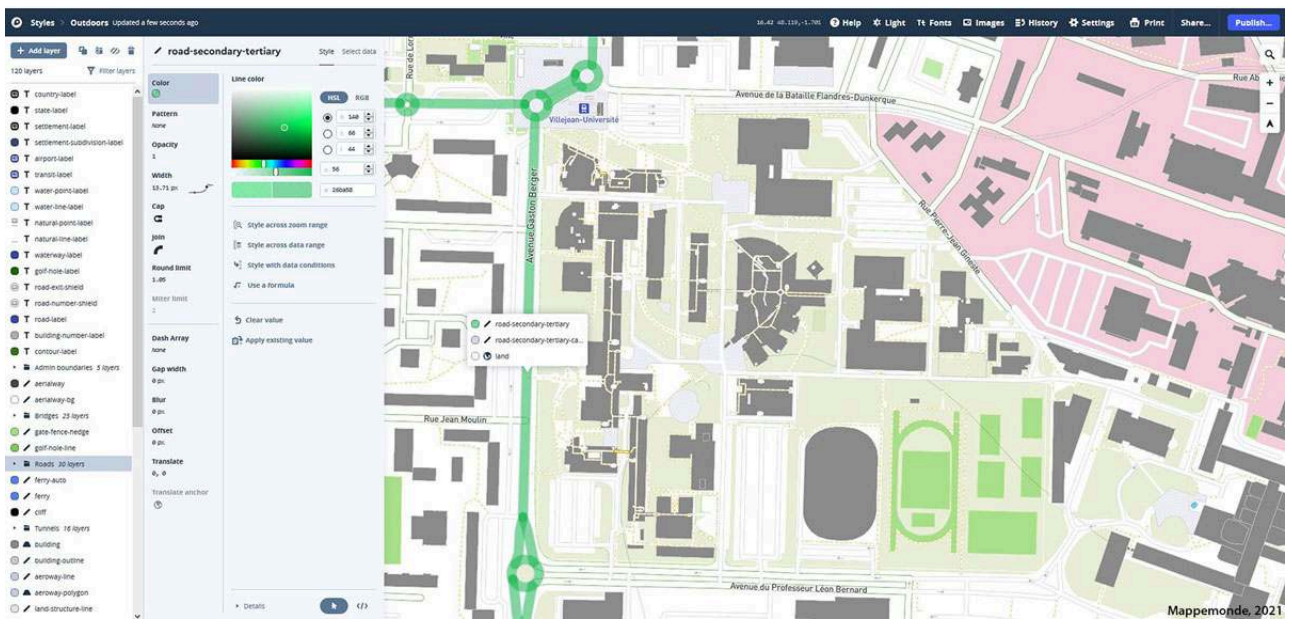

https://www.mapbox.com/mapbox-studio/

Les enjeux autour de la personnalisation des fonds de cartes renvoient à la fois à des questionnements sur les données à afficher (quels éléments afficher en fonction du niveau de zoom) comme à leurs caractéristiques de mise en forme (couleur, épaisseur, opacité, forme et typographie des étiquettes) (Dubrava, 2017; Beukelaar, 2018). Ce paramétrage, qui relève de décisions prises en amont lors de la génération des tuiles (dans un serveur ou via un service interfacé), constitue une étape importante dans la conception des cartes en ligne. L'un des chantiers les plus dynamiques actuellement 
porte sur les ombrages et plus généralement autour de l'intégration des courbes de niveau pour représenter de manière plus réaliste la topographie. La question de l'expressivité des fonds de carte constitue une autre piste particulièrement intéressante pour améliorer l'adéquation entre les représentations et les usages associés (Christophe, 2017). L'objectif est ici d'explorer des styles de fonds de carte plus expressifs, voire artistiques, afin de faciliter la transmission de messages cartographiques et rendre les cartes plus efficaces.

Il est intéressant d'observer qu'avec cette nouvelle génération d'outils, qui permettent en quelques clics de personnaliser à l'infini les fonds de carte, on assiste paradoxalement à une certaine normalisation des styles disponibles sur le géoweb. Le style «Plan» popularisé depuis 2005 par Google Maps est devenu un standard, largement imité par la majorité des fournisseurs de fonds de carte. L'analyse de Dubrava (2017) sur ce sujet montre par exemple que les utilisateurs préfèrent largement un style similaire à celui de la firme de Mountain View qu'à tout autre style. La personnalisation des fonds de carte trouve également aujourd'hui sa place au niveau du tracé des frontières qui font l'objet de litiges. Ces dernières peuvent en effet s'adapter automatiquement à la nationalité des usagers via l'adresse IP, comme c'est le cas dans Google Maps. Le service WorldView ${ }^{24}$ de Mapbox propose dans cet esprit un service de fonds de carte en tuiles vectorielles où les tracés des frontières proposent trois visions géopolitiques (États-Unis, Chine et Inde) en fonction du public visé.

Malgré des potentialités intéressantes tant sur le plan de l'affichage, de la personnalisation que de l'interaction, les cartes en ligne basées sur les tuiles vectorielles restent (encore) minoritaires par rapport aux cartes basées sur les tuiles raster (Netek et al., 2020). Toutefois les pratiques évoluent très rapidement, les grands acteurs du géospatial ont largement pris le virage des tuiles vectorielles, et de leur côté, les acteurs publics commencent à explorer et à s'approprier cette technologie. Le principal frein à une plus grande utilisation s'explique en partie par l'existence d'un verrou technique. La technologie des tuiles vectorielles nécessite en effet des compétences en programmation pour à la fois mettre en place des serveurs de tuiles, mobiliser ces flux d'un type particulier et configurer la personnalisation. Cependant, plusieurs services en ligne interfacés, commerciaux et open source, permettent de mettre en place rapidement des fonds de carte comme des services de tuiles vectorielles sans ligne de code (Mapbox, ESRI, Google, Jawg, etc.) Et, de manière générale, les outils de cartographie en ligne commencent à intégrer les tuiles vectorielles au sein des environnements existants ${ }^{25}$. Cette approche de la cartographie en ligne n'en est qu'à ses prémices, et au regard des enjeux relatifs aux données volumineuses, cette technologie constitue l'une des voies les plus dynamiques qui devrait se généraliser dans les années à venir.

\section{L'agrégation des données spatiales volumineuses comme enjeu de géovisualisation}

Au-delà de la diffusion des données spatiales, l'un des principaux défis pour la cartographie en ligne est de proposer des solutions pour représenter spatialement des milliers, voire des millions d'objets géographiques de manière fluide, lisible et compréhensible (Gaffuri, 2012). Cette problématique concerne essentiellement les données ponctuelles, lesquelles sont toujours plus nombreuses et diversifiées sur le 
géoweb. Ce paradigme de la "punaise cartographique» (Singleton et Brunsdon, 2014) est aujourd'hui largement ancré dans les pratiques comme dans l'imaginaire collectif et constitue un nouveau vocabulaire graphique de la cartographie en ligne.

Les méthodes conventionnelles de représentations cartographiques mobilisant des variables visuelles de taille, de couleur ou de forme ne permettent pas de répondre à ces nouveaux besoins de géovisualisation en raison des volumes de données (Netek et al., 2019). L'affichage de milliers de points sous la forme de marqueurs pose le problème du chevauchement des données qui limite leur identification et de manière générale diminue les capacités cognitives d'interprétation (Ježek et al., 2017). Se pose en plus la problématique des performances de rendu graphique qui induit de repenser les modes de représentation pour minimiser la taille des données transmises et ainsi fluidifier l'affichage (Fürhoff, 2019 ; Netek et al., 2019).

En réponse à ces besoins, plusieurs types d'approches, lesquelles reposent sur la réduction de la complexité des données par des méthodes d'agrégation spatiale, ont fait leur apparition ces dernières années. L'objectif est ici de procéder à une simplification de l'information par le regroupement ou l'interpolation spatiale des données. L'utilisation grandissante de l'agrégation sous la forme de carte de chaleur (heatmap), de regroupement sous forme de "grappes » (cluster) et de cartes en grilles (grid) occupe une place importante dans le régime cartographique du géoweb (Mericskay, 2016). Issues des techniques et des méthodes de visualisation d'informations (infovis), ces approches permettent d'interpoler, d'agréger et de résumer de gros volumes de données pour faciliter leur interprétation (Fekete et Plaisant, 2002 ; Keim et al., 2008 ; Slocum et al, 2009).

Comme le soulignent Korpi et Ahonen-Rainio (2013), les applications de cartographie en ligne possèdent à la fois des caractéristiques relevant de la cartographie et de la visualisation d'informations. En ce sens, les méthodes des deux disciplines doivent être prises en compte dans leur construction. De plus, la possibilité de changer d'échelle induit de repenser les modalités comme les méthodes d'agrégation spatiale sous des formes dynamiques pour aider les utilisateurs à décider où ils souhaitent se déplacer et zoomer pour l'exploration des données (Gaffuri, 2011 ; Ježek et al., 2017).

\section{Le regroupement sous la forme de clusters comme méthode privilégiée}

Très populaire auprès des informaticiens et des développeurs Web, le regroupement spatial de marqueurs sous forme de clusters, tient une place importante dans la visualisation de données spatiales sur le Web (Fürhoff, 2019). Cette approche basée sur l'analyse des distances et des densités d'un ensemble de points permet de rendre plus lisible une carte saturée de points par leur regroupement en grappes homogènes et l'affichage de leur nombre (Meier, 2016). Très utilisée dans le domaine de l'analyse des données, cette méthode renvoie à un ensemble de techniques de partitionnement aux approches diversifiées ${ }^{26}$ (Meert, 2006 ; Rezaei et Franti, 2018). Avec les environnements matériels et les navigateurs de plus en plus puissants, il est possible de traiter des millions de marqueurs spatiaux en temps réel et de proposer côté client des temps de chargement d'affichage très rapides (Netek et al., 2019).

L'approche par cluster sur le géoweb renvoie à la fois à une dimension dynamique et interactive. Ainsi il est possible à une échelle régionale ou nationale de visualiser la

Mappemonde, 131 | 2021 
concentration de points par leur agrégation et à une échelle locale de pouvoir visualiser les données dans leur individualité et d'interroger leurs caractéristiques attributaires (Meier, 2016). Ce mode de représentation permet ainsi de répondre à un enjeu important en matière de visualisation de données, à savoir la possibilité de proposer une vue globale, de zoomer et d'obtenir davantage d'informations à la demande (Shneiderman, 1996).

Sur le plan de l'interaction, il existe plusieurs manières d'interagir avec les clusters et les données qu'ils agrègent. La forme d'interaction systématique repose sur un changement dynamique où les clusters s'adaptent en fonction du niveau zoom. On trouve également des formes plus poussées qui permettent par exemple d'afficher à la volée l'étendue spatiale des clusters en survolant ou en cliquant sur un cluster. En complément, en cliquant sur un cluster, il est également possible d'effectuer un zoom (automatique) sur l'emprise de ce cluster pour afficher le regroupement à une autre échelle, voire faire apparaître, à la volée, les données de manière individuelle (figure 4).

Figure 4. (a) Cluster d'adresses avec affichage de l'emprise du regroupement au survol) ; (b) Cluster d'adresses avec affichage des données individuelles au clic ; (c) Cluster des tremblements de terre avec affichage des données individuelles au survol

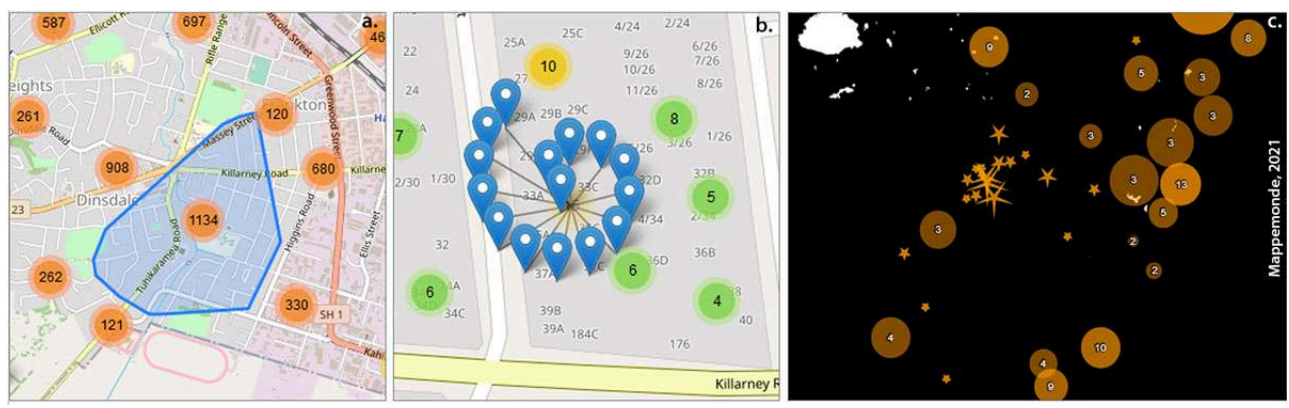

(a) Leaflet : http://leaflet.github.io/Leaflet.markercluster/example/marker-clustering-realworld 50000.html ; (b) Leaflet : http://leaflet.github.io/Leaflet.markercluster/example/marker-clusteringrealworld.50000.html ; (c) OpenLayers : https://openlayers.org/en/latest/examples/earthquakeclusters.html

29 Au niveau du rendu graphique, la tendance est à la complexification des formes de représentation des clusters (figure 5). Dans une optique de gain en efficacité, l'ajout de variables visuelles (taille, forme, couleur) aux clusters permet d'aider les utilisateurs à identifier, classer et comparer les entités sur la carte (Meier, 2016; Fürhoff, 2019). La forme la plus classique consiste à introduire une nouvelle entité visuelle au sein de la carte qui différencie les clusters (le plus souvent des cercles) des marqueurs uniques et qui sont agrémentés d'une étiquette indiquant le nombre de marqueurs agrégés. D'autres méthodes permettent de combiner une variation de taille et de couleur dans la représentation des clusters pour mieux différencier le volume de marqueurs regroupés. Il est également possible d'intégrer des variables quantitatives associées aux marqueurs qui peuvent elles-mêmes être prises en compte dans l'agrégation (somme, moyenne) et affichées au sein des étiquettes. Enfin, des méthodes plus complexes commencent à émerger, comme celles qui permettent de produire à la volée des clusters basés sur des diagrammes proportionnels. Cette approche est particulièrement intéressante, car elle combine à la fois la représentation du volume de marqueurs regroupés et les attributs des objets sous la forme de graphiques en secteurs. 
Figure 5. (a) Cluster des horodateurs de Paris (Ville de Paris) ; (b) Cluster d'adresses (Leaflet) ; (c) Cluster des tremblements de terre aux États-Unis (Mapbox); (d) Cluster/diagramme des sites de production d'électricité (Mapbox)
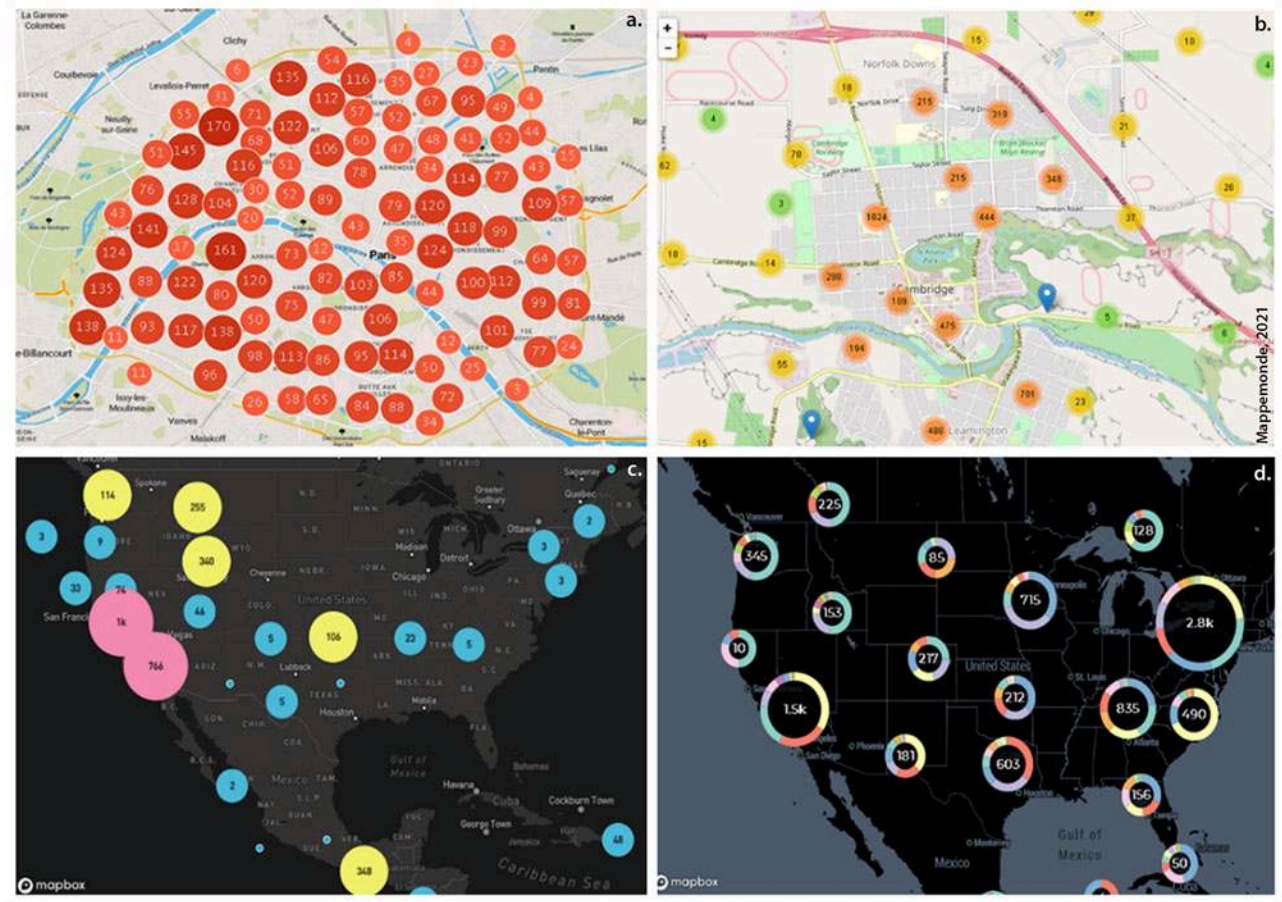

(a) https://opendata.paris.fr/explore/dataset/horodateurs-mobiliers/map/?

disjunctive.alim\&disjunctive.arrondt\&disjunctive.modele\&disjunctive.regime\&disjunctive.tarif\&disjunctive.zoneres\&disjunctive.tarifhor\&locatio (b) http://leaflet.github.io/Leaflet.markercluster/example/marker-clustering-realworld.50000.html ; (c) https://docs.mapbox.com/mapbox-gl-js/example/cluster/ ; (d) https://blog.mapbox.com/clusteringproperties-with-mapbox-and-html-markers-bb353c8662ba?gi=34f8e5771228

\section{L'agrégation basée sur des carroyages}

L'agrégation spatiale de données basées sur des grilles (grid), aussi appelées binning (regroupement dans des boîtes) constitue une autre technique de plus en plus mobilisée sur le géoweb. Cette méthode, bien connue en analyse spatiale, propose de synthétiser et de représenter des ensembles de données géographiques (ponctuelles) au sein d'unités spatiales surfaciques régulières et homogènes (Amini et al., 2011 ; Kiefer, 2015). Ce mode de partition particulier de l'espace, dont l'unité de base est la maille, peut prendre différentes formes au niveau des géométries mobilisées (carreaux, mailles hexagonales, losanges ou rectangles). En s'affranchissant à la fois des découpages administratifs et de la structuration des territoires (hydrologie, relief, voirie, etc.), cette approche basée sur une discrétisation de l'espace permet de mettre en évidence des tendances de répartition spatiale d'un phénomène. Visuellement, la représentation sous forme de grille régulière offre une lecture plus aisée des informations (Kiefer, 2015).

31 La valeur ajoutée de cette méthode réside dans la possibilité d'exécuter un ensemble de fonctions pour résumer statistiquement leurs caractéristiques (dénombrement, moyenne, somme, minimum, etc.). En complément, et de manière similaire aux clusters, l'une des innovations les plus intéressantes se situe au niveau de la dimension dynamique des carroyages et, par ricochet, des agrégations opérées. Générées à la volée, la granularité comme la forme des mailles peuvent s'adapter à l'échelle de 
visualisation et ainsi proposer des grilles selon des résolutions grossières comme très fines, en fonction des données ou des objectifs. De plus, les variables analysées et les méthodes d'agrégation statistique peuvent également être modifiées à la volée comme l'illustre la figure 6. Selon cette perspective, cette méthode est particulièrement pertinente pour l'exploration de données spatiales caractérisées par des attributs numériques. Toutefois, le paramétrage de la taille comme de la forme des grilles n'est pas toujours personnalisable, voire, dans certains cas, il n'est pas possible de connaitre leurs dimensions limitant ainsi leur analyse.

Figure 6. (a) Implantation ponctuelle des 34580 ventes d'appartements à Paris en 2017 ; (b) Nombre total de ventes d'appartements par carreau; (c) Somme des valeurs de vente d'appartements par carreau ; (d) Moyenne des valeurs de vente au $\mathrm{m}^{2}$ des appartements par carreau
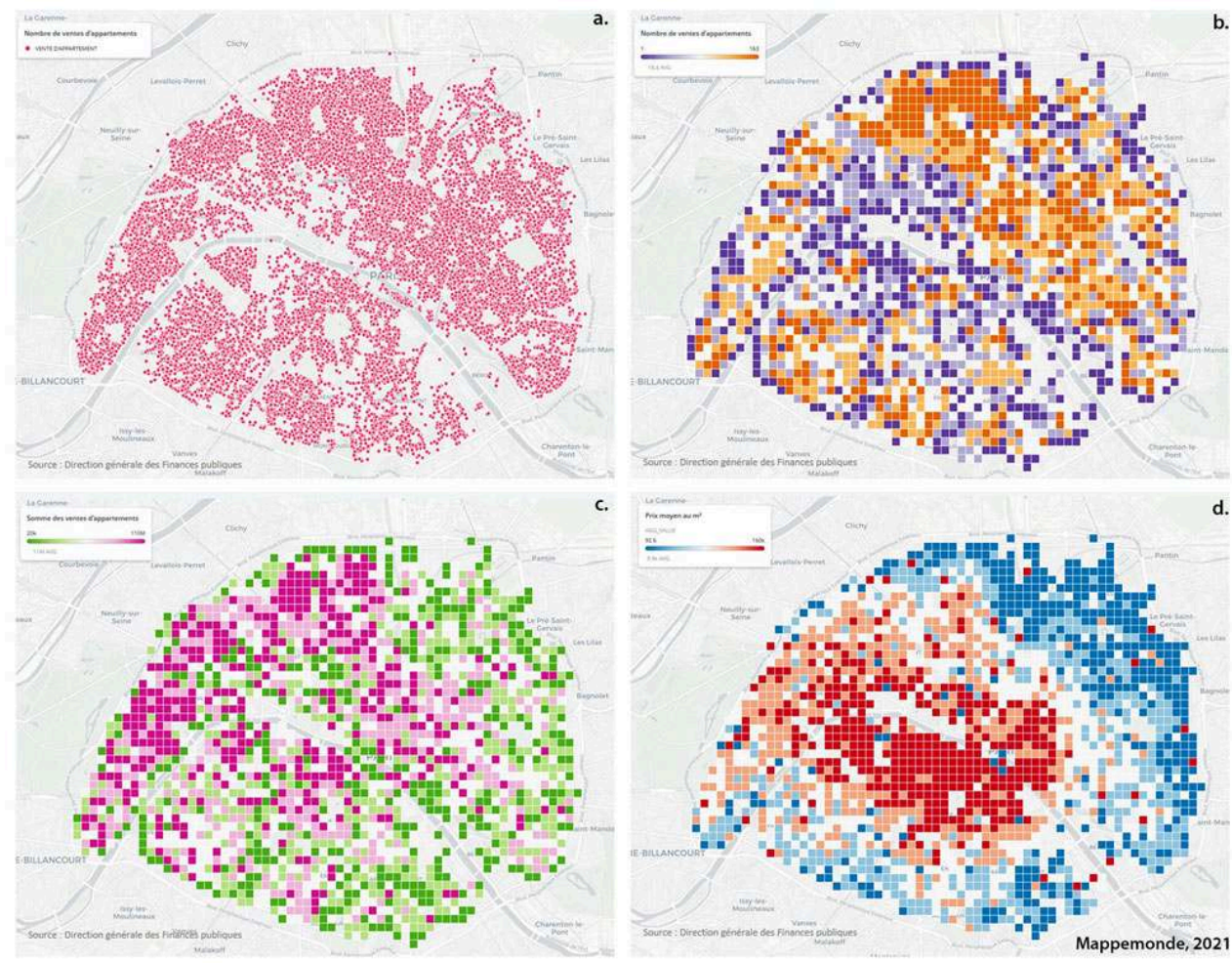

B. Mericskay

Assez peu présente comme manière de représenter des données agrégées au sein d'une grille, la mobilisation de cercles proportionnels en remplacement des aplats de couleurs constitue une alternative intéressante. Basée sur une variation de taille de figurés placés au centre des mailles, cette approche est particulièrement pertinente dans la mesure où elle permet d'appliquer à l'agrégation un mode de représentation plus adapté à des valeurs de stock et plus familier pour les spécialistes (figure 7). 
Figure 7. Agrégation des populations des grandes villes du monde au sein d'un carroyage

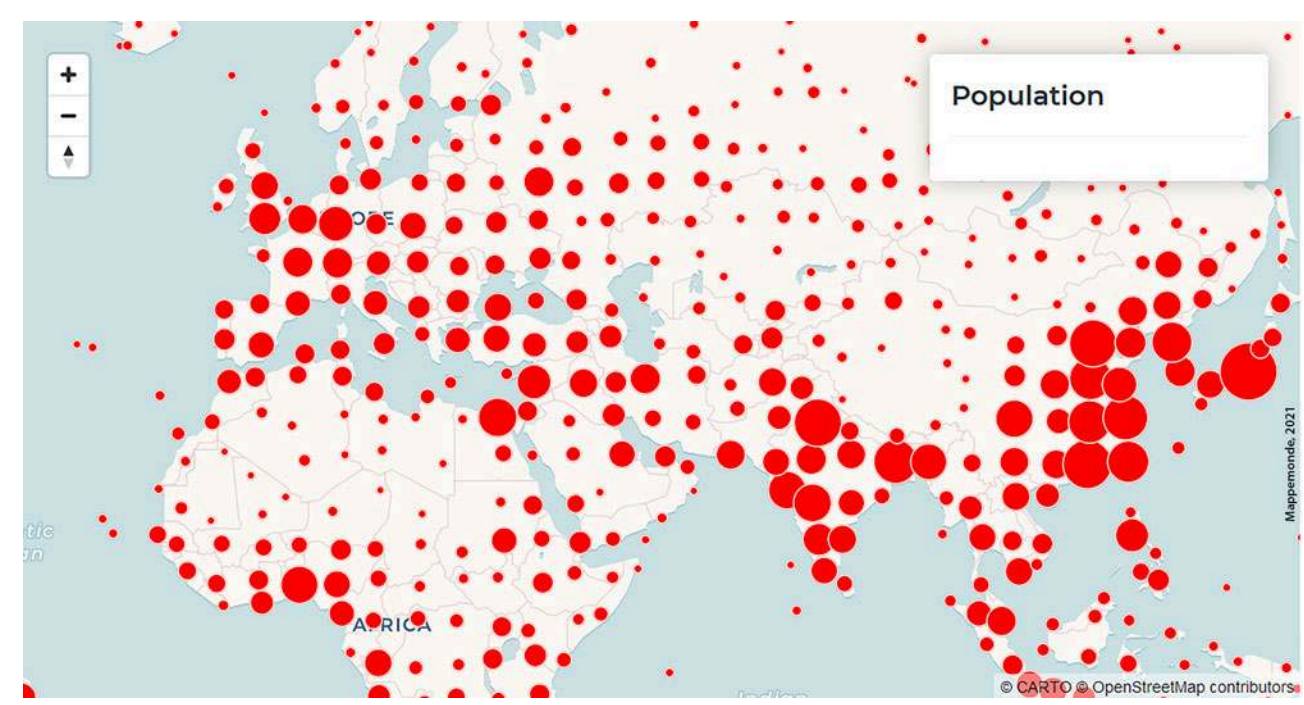

CARTO : https://carto.com/developers/carto-vl/v0.10.0/guides/aggregations/

\section{Les cartes de chaleur (heatmap)}

33 À la différence des approches par cluster et carroyage qui permettent une représentation discrète des données, les cartes de chaleur (heatmap) proposent de leur côté une représentation continue d'un ensemble de données ponctuelles par l'estimation des densités de noyau (Ježek et al., 2017). Une carte de chaleur est une grille raster pour laquelle on attribue à chaque pixel une valeur de mesure correspondant à la densité de points dans et autour de chaque pixel.

Les heatmaps fournissent un aperçu visuel intuitif de l'intensité d'un phénomène par rapport à une région spatiale (Trame et Kessler, 2011). Il est ainsi possible d'identifier en un coup d'œil les zones à forte densité (les points chauds), la distribution ainsi que l'organisation spatiale des concentrations d'un ensemble de données comme l'illustrent les exemples de la figure 8 (DeBoer, 2015 ; Netek et al., 2019). Sur le géoweb, les cartes de chaleur sont dynamiques et s'adaptent à l'échelle de visualisation. Toutefois, les formes d'interactions se limitent au simple changement de zoom. De plus, à la différence des approches d'agrégation par cluster ou maillage, ce mode représentation ne permet pas visualiser les données de manière individuelle et de venir précisément quantifier la quantité de données regroupées. 
Figure 8. (a) Heatmap de tremblements de terre (Leaflet) ; (b) Heatmap de tremblements de terre (OpenLayers) ; (c) Heatmap de tremblements de terre (Mapbox) ; (d) Heatmap de points aléatoires (Google Maps)
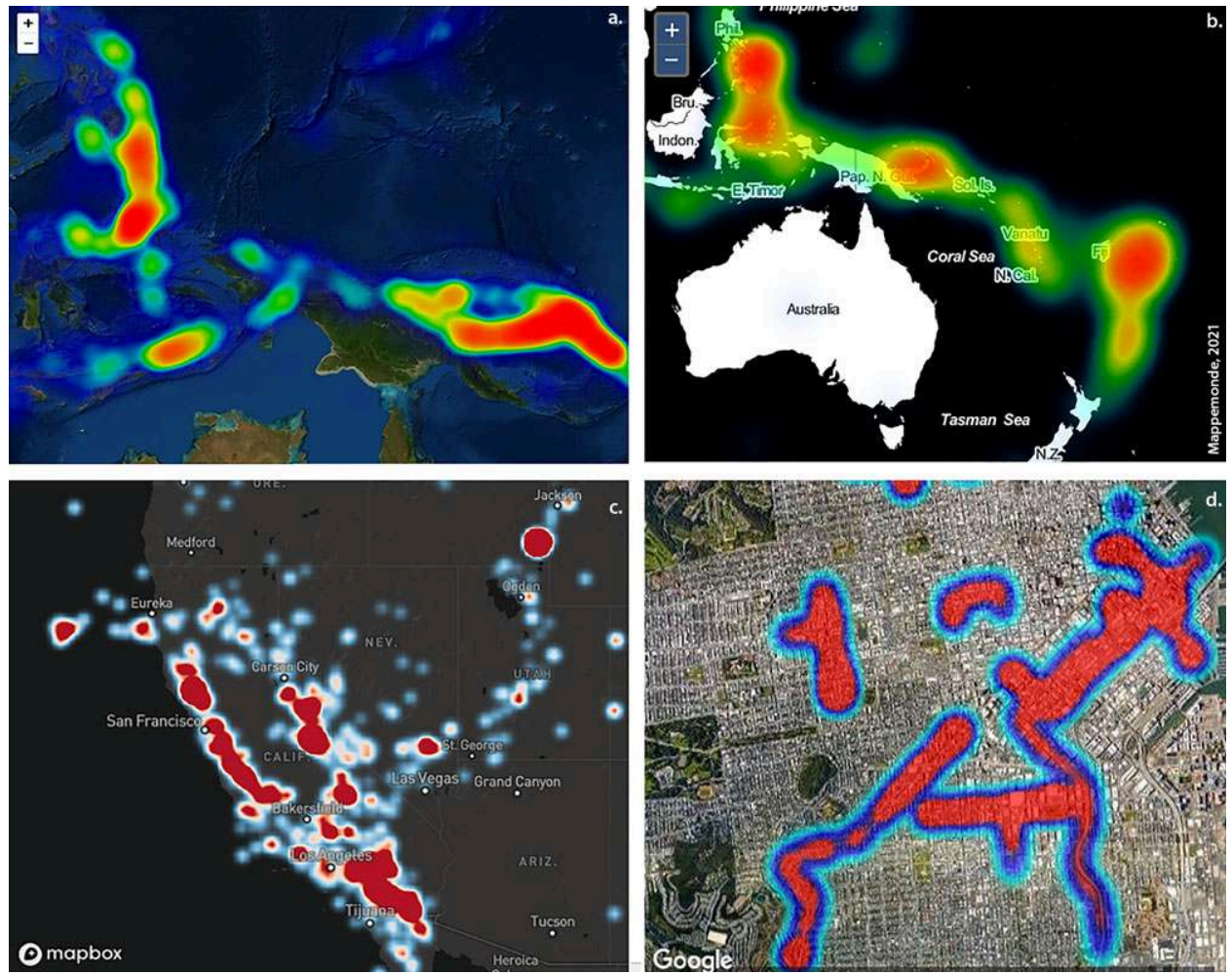

(a) Leaflet : https://www.datavis.fr/index.php?page=leaflet-heatmap ; (b) OpenLayers : https:// openlayers.org/en/latest/examples/heatmap-earthquakes.html ; (c) Mapbox : https:// docs.mapbox.com/mapbox-gl-js/example/heatmap-layer/ ; (d) Google Maps : https:// developers.google.com/maps/documentation/javascript/examples/layer-heatmap

Ce mode de représentation très apprécié sur le géoweb présente d'autres limites, notamment au niveau de la configuration du rendu visuel des cartes. À la différence des outils SIG où l'utilisateur peut choisir différentes méthodes et les paramétrer à sa guise, au sein des outils de cartographie en ligne assez peu de paramètres peuvent être configurés pour produire des cartes de chaleur claires et pertinentes. Sur le plan de la construction de la carte, il est possible de paramétrer le rayon dont la propriété affecte la précision de la carte en définissant l'influence de chaque point en pixels. En complément, certains environnements autorisent l'intégration d'une valeur de pondération (basée sur un attribut des objets) pour apporter plus de précision au rendu cartographique. Bien que les cartes de chaleur soient visuellement efficaces, les représentations proposées peuvent être incorrectes, voire trompeuses. Cette technique doit être utilisée avec prudence, car il n'y a souvent peu d'indications sur la façon ces cartes sont construites (DeBoer, 2015).

Au niveau de symbologie, la gamme de ton, le nuancier de couleur, l'intensité et l'opacité des cartes de chaleur sont la plupart du temps personnalisables. Mais, de manière générale, ce mode de représentation, basé sur un raster, ne propose pas une grande variété de mise en forme dans la mesure où seule la variable visuelle de couleur est paramétrable. (Meier et al., 2014).

Le recours aux heatmap, aux carroyages comme aux clusters constitue un pan important de la sémiologie graphique de la cartographie en ligne. Au-delà d'opportunités 
techniques intéressantes pour représenter de gros volumes de données spatiales, la question de l'efficacité visuelle en matière de compréhension du message cartographique se pose. Les notions de lisibilité et d'utilisabilité de ces modes de représentation qui renvoient à des problèmes de perception, de cognition et d'interaction sont encore peu explorées. L'étude de Meier (2016) sur l'évaluation de ces méthodes auprès d'un groupe de lecteurs met notamment en avant qu'elles sont attractives visuellement, mais assez peu efficaces sur le plan de la compréhension. Les sujets éprouvent en effet des difficultés à décoder et comprendre ces modes de représentation, notamment les clusters.

De plus, le rendu visuel de ces approches manque souvent de sens dans la mesure où les agrégations proposées sont le résultat de traitements automatisés où les cartographes ont assez peu la main sur le paramétrage des agrégations et la symbologie (Furhoff, 2019). De fait, les concepteurs de ces cartes d'un type particulier, souvent eux-mêmes peu spécialistes de l'analyse spatiale, sont bien souvent contraints de mobiliser des méthodes comme des modes de représentation préétablis dont ils ignorent les logiques sous-jacentes. Enfin il apparaît qu'avec la généralisation de ces méthodes, on assiste à une certaine standardisation des cartes en ligne pour la représentation de données massives.

Toutefois, il ne faut pas perdre de vue que l'objectif de ce type de cartes en ligne n'est pas de fournir des représentations figées à des fins d'analyse, mais davantage de proposer des interfaces cartographiques pour explorer de manière dynamique et interactive des données spatiales. De plus, ces approches basées sur l'agrégation ne visent pas à représenter des données géographiques selon une approche individuelle, mais bien à faire apparaitre des structures spatiales, des logiques de concentration dans une optique multi-échelle. Aucune des méthodes présentées ici n'est parfaite, mais en fonction des objectifs et du type de données à représenter, chacune possède des avantages et des inconvénients résumés dans le tableau 1.

Tableau 1. Synthèse des trois méthodes d'agrégation spatiale

\begin{tabular}{|l|l|l|l|}
\hline & Heatmap & Cluster & Carroyage \\
\hline Paramétrage de l'agrégation & -+ & -+ & + \\
\hline Variable de pondération & + & -+ & + \\
\hline Agrégation statistique & - & -+ & + \\
\hline Efficacité visuelle & + & -+ & + \\
\hline Visualisation des structures spatiales & + & - & -+ \\
\hline Quantification du volume de données & -+ & + & + \\
\hline Visualisation individuelle des données & - & + & - \\
\hline Personnalisation de la symbologie & -+ & + & + \\
\hline
\end{tabular}


Interaction avec les données

B. Mericskay

\section{De nouveaux modes de géovisualisation en trois dimensions}

\section{Le WebGL comme innovation technique}

Le développement du WebGL comme standard pour la visualisation 3D sur le Web est une autre innovation technique importante dans les formes que prennent les cartes en ligne aujourd'hui (Krämer et Gutbell, 2015 ; Farkas, 2017). Cette spécification pour les navigateurs Web permet d'afficher, de créer et de gérer dynamiquement des éléments graphiques complexes en 3D au sein d'un navigateur Web sans utilisation de modules complémentaires (Farkas, 2019). Basé sur le langage JavaScript ${ }^{27}$, un système d'API et l'accélération matérielle ${ }^{28}$, le WebGL est porteur d'importants enjeux dans l'utilisation de la 3D au sein des cartes sur le Web.

41 Google a été l'un des premiers acteurs à se saisir des potentialités de cette innovation technique pour la cartographie en ligne. Dès 2010, le service Google Maps a intégré ces nouvelles spécifications pour de meilleures performances d'affichage, des graphismes en 3D plus précis, des transitions plus fluides et la possibilité d'incliner et de changer d'orientation. Depuis, plusieurs bibliothèques JavaScript dédiées à la cartographie en WebGL ont fait leur apparition comme Cesium ${ }^{29}$, Tangram ${ }^{30}$ ou MapboxGL ${ }^{31}$. Récemment, plusieurs acteurs du géospatial ont pris le virage du WebGL en développant leurs propres bibliothèques logicielles afin de tirer parti de ces avancées techniques à l'image d'ESRI (ArcGIS API for JavaScript ${ }^{32}$ ), de Uber (DeckGL ${ }^{33}$ ), de HERE (harp.gl ${ }^{34}$ ) ou de l'IGN (iTowns ${ }^{35}$ ).

42 Combiné aux tuiles vectorielles, le WebGL transforme les cartes en ligne notamment en s'affranchissant de la classique vue planisphérique orientée nord. Il est désormais possible, au-delà des fonctions classiques de déplacement et de zoom, de faire pivoter et d'incliner les cartes. Cette évolution en matière d'interaction avec la vue de la carte ouvre la voie à de nouvelles façons d'envisager les cartes en ligne et les manières de représenter des données spatiales à travers notamment la mobilisation de l'extrusion.

La récente version vectorielle du géoportail fédéral suisse (figure 9) est un bon démonstrateur pour illustrer à quoi peut ressembler la nouvelle génération d'application de cartographie en ligne. Basée sur le WebGL et les tuiles vectorielles, cette application permet d'explorer le territoire helvétique à travers son relief et 70 millions d'objets en 3D (infrastructures, routes, bâtiments, végétation...). Cette forme de portail cartographique Web n'en est encore qu'à ses débuts, mais augure une nouvelle génération d'outils en ligne (Nguyen et al., 2015 ; Lafrance et al., 2019). 
Figure 9. Interface du géoportail fédéral suisse (geo.admin.ch)

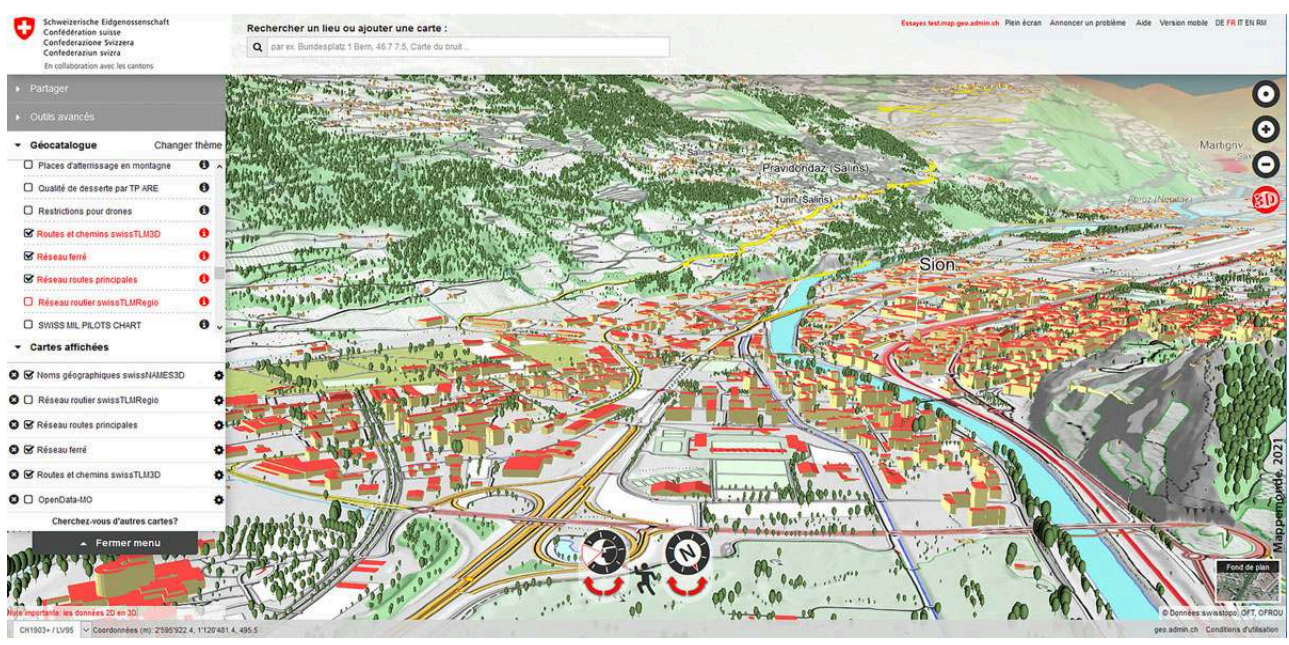

https://map.geo.admin.ch/?

layers $=$ ch.swisstopo.swissnames3d\&lon=8.24528\&lat=46.04722\&elevation=87928\&heading $=360.000 \&$ pitch $=-44.188 \&$ lang=en\&topic $=e c h \& b c$ farbe

\section{Vers de nouveaux modes de géovisualisation en 3D}

Le passage à la troisième dimension induit de nouvelles formes de perception des données, qui font appel à des capacités d'interprétation différentes de celles des cartes en deux dimensions (Ware et Plumlee, 2005). Les possibilités d'extrusion 3D de données diversifiées (bâtiments, statistiques, flux, etc.) autorisent, de leur côté, de nouvelles formes de géovisualisation pour l'exploration de données territoriales et l'analyse de phénomènes socio-spatiaux.

\section{La géovisualisation de bâtiments en 3D}

Les référentiels vectoriels de bâtiments, davantage complets, précis géométriquement et sémantiquement (nombre d'étages, hauteur...) sont de plus en plus représentés en trois dimensions sur le géoweb. Avec le WebGL, il est assez simple d'extruder la hauteur des bâtiments pour donner à voir de manière plus réaliste les volumétries des villes. L'idée est de proposer des référentiels cartographiques en ligne en 3D, où l'utilisateur peut se déplacer et y superposer d'autres données. Au niveau de la symbologie, de plus en plus de géovisualisations proposent d'appliquer des catégorisations ou des graduations de couleurs aux bâtiments pour représenter des informations comme la hauteur, le nombre d'étages, l'âge ou le zonage en vigueur (figure 10).

Il faut cependant préciser que tous les types de constructions, comme les bâtiments à l'architecture complexe, ne peuvent pas être fidèlement représentés par de simples extrusions. Une représentation plus réaliste passe par l'utilisation de vrais modèles 3D, encore difficilement mobilisables au sein des environnements en ligne. De plus, la superposition d'objets géographiques en $2 \mathrm{D}$ et en $3 \mathrm{D}$ au sein d'une même vue peut poser des problèmes d'affichage et de lisibilité. Afin de favoriser le développement de cette forme de cartographie, un format dédié aux tuiles 3D, intitulé 3D Tiles $^{36} \mathrm{a}$ récemment émergé. Portée par l'Open Geospatial Consortium, cette spécification permet 
d'intégrer des données 3D hétérogènes et volumineuses ( $\mathrm{BIM}^{37}$, maquette urbaine, données LIDAR) au sein des environnements Web.

Figure 10. (a) Visualiseur 3D de données d'OpenStreetMap ; (b) Extrusion du bâti de la ville de Paris ; (c) Cartographie intérieure de l'université du Michigan ; (d) Volumétrie 3D du bâti et zonage à Vancouver
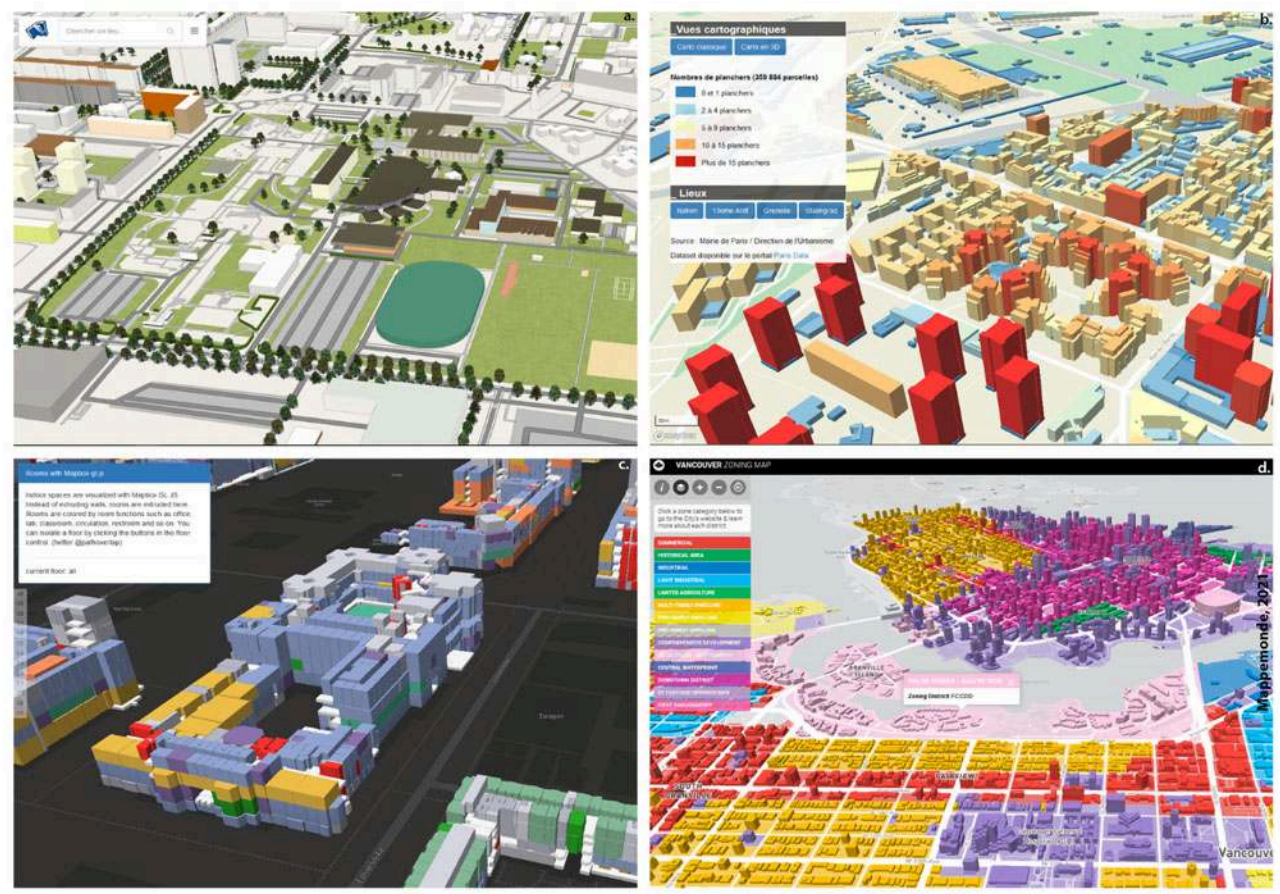

(a) F4Map : https://demo.f4map.com/\#camera.theta=0.9 ; (b) Boris Mericskay : https://sitesformations.univ-rennes2.fr/mastersigat/WebMaps/Parisbati.html ; (c) Yongha Hwang : http://wwwpersonal.umich.edu/ yonghah/rooms3d/ ; (d) Robert White : http://maps.nicholsonroad.com/zones/

\section{Le réinvestissement de la cartographie thématique en trois dimensions}

Les cartes thématiques en 3D, qui permettent la représentation tridimensionnelle de données statistiques connaissent de leur côté un renouvellement des usages. Exploré depuis longtemps par les cartographes (Jenks, 1963 ; Franklin et Lewis, 1978), ce mode de représentation traditionnellement difficile à mobiliser fait aujourd'hui l'objet de développements du fait d'avancées techniques autour de l'affichage en 3D. Le principe est simple, extruder des entités polygonales en 2D (contours administratifs, carroyage) en fonction d'une variable statistique (population, nombre d'accidents, résultats électoraux, etc.). Chaque polygone devient ainsi un prisme de hauteur, proportionnelle à la valeur d'un attribut (Rase, 2009). Cette technique de conception cartographique présente l'avantage de donner à voir autrement des différences de proportionnalité entre unités spatiales selon une approche où la hauteur des unités spatiales permet de les différencier (figure 11). 
Figure 11. (a) Nombre d'individus par carreau en Occitanie ; (b) Accidentologie au Royaume-Uni ; (c) Nombre d'individus par census à New York ; (d) Population et densité de population par comté américain
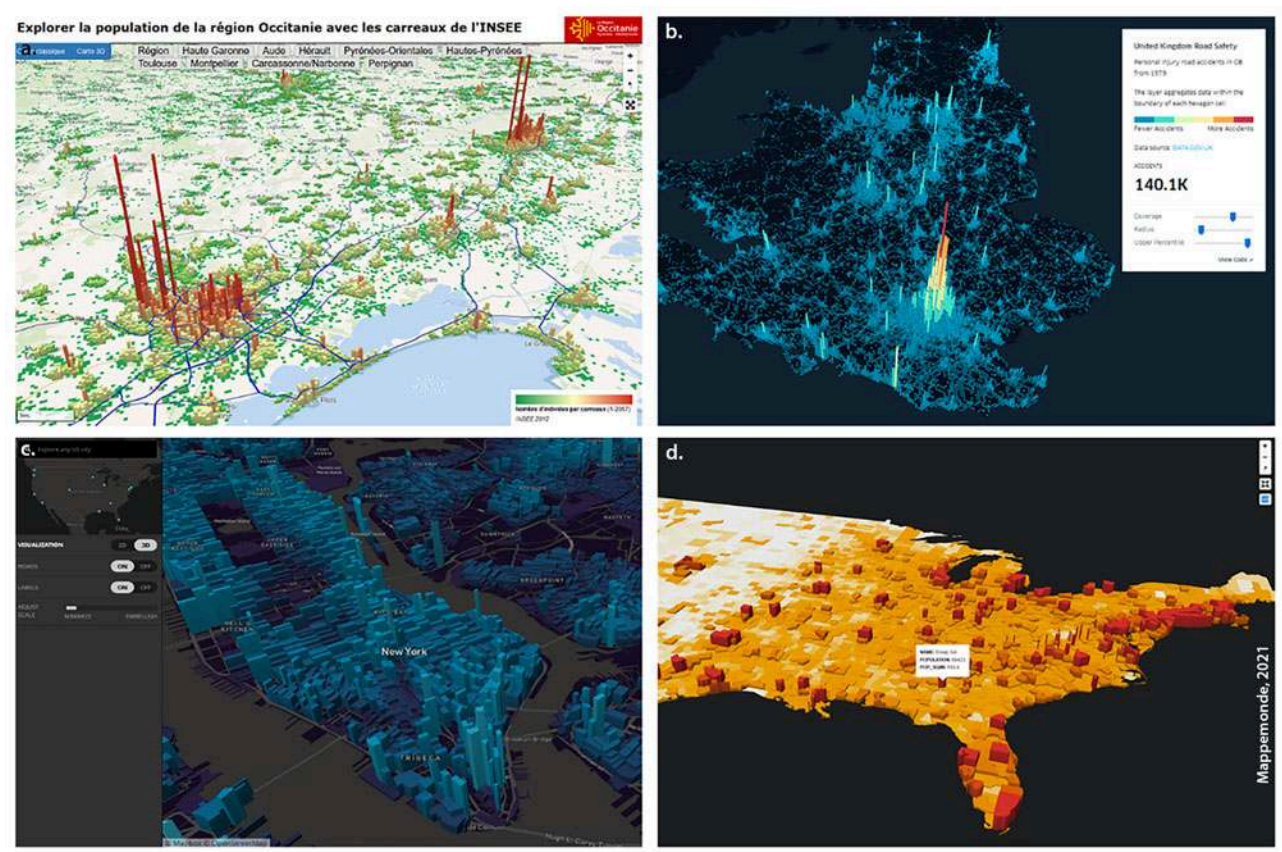

(a) Boris Mericskay : https://sites-formations.univ-rennes2.fr/mastersigat/WebMaps/

Extrusion_Occitanie.htm ; (b) DeckGL https://deck.gl/examples/hexagon-layer/ ; (c) Mapbox : https:// labs.mapbox.com/bites/00273; (d) ovrdc.org : https://ovrdc.github.io/gis-tutorials/mapbox/05-2-

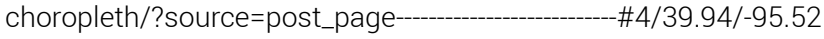

Cette approche est particulièrement intéressante en cartographie thématique, car elle permet une représentation alternative des variables de stock, lesquelles sont le plus souvent symbolisées par la variation de taille d'un symbole en deux dimensions. Dans le cadre de l'extrusion en 3D d'un polygone, la forme de chaque unité est conservée et la hauteur fait office de variable visuelle (figure 11). Il est ainsi possible au niveau de la symbologie de combiner deux variables visuelles pour une même unité spatiale (couleur et taille). Cette perspective est particulièrement intéressante pour venir renforcer le message (redondance visuelle de l'attribut par l'extrusion et la couleur) et favoriser la différenciation entre entités spatiales d'une même classe (Slocum et al., 2009 ; Pachinger et al., 2016). Ce mode, basé sur deux variables visuelles, peut également être mobilisé pour la représentation de deux attributs différents dans une optique d'enrichissement de la carte, avec toutefois un risque de complexification de lecture.

Autre dimension intéressante, la possibilité de mobiliser ce mode de représentation à de petites échelles afin de produire une vision d'un relief qui fait appel à des capacités d'interprétation plus basiques que la lecture d'une carte en deux dimensions (Jégou, 2007). Les projets Human Terrain ${ }^{38}$ et Population Mountain ${ }^{39}$, développés par des data journalists, proposent selon cette perspective d'explorer, à l'échelle du monde, la répartition de la population dans le temps et dans l'espace au sein d'un carroyage en 3D de $250 \mathrm{~m}$. Ce type de carte qui dessine des "paysages» constitués de monts et de montagnes permet de rendre compte de l'intensité des phénomènes représentés par la hauteur des « pics» (Lambert et Zanin, 2016).

Cependant, ce mode de représentation encore peu mobilisé s'accompagne de certaines limites sur le plan de la lecture et de la compréhension. L'étude menée par Popelka 
(2018) met par exemple en évidence que les cartes choroplèthes en 3D permettent une meilleure compréhension de l'information transmise que les cartes choroplèthes en 2D. Toutefois, les répondants ont tendance à prendre plus de temps pour interpréter ces informations. Ce constat est aussi partagé dans l'étude de Stewart et Kennelly (2019) sur les cartes en prismes ombragées, où les utilisateurs ont tendance à davantage décortiquer les ombres que les aplats de couleurs pour en tirer de l'information. De son côté, White (2012) met également en avant que la lecture de cartes thématiques en 3D est plus exigeante et moins naturelle que les cartes choroplèthes en 2D.

\section{Vers une cartographie des flux en 3D}

51 Les possibilités offertes par le WebGL renouvellent également les manières de représenter spatialement les interactions entre des couples de lieux. Sur la base d'une carte de liens en 2D, il est désormais plus facile de mobiliser des arcs en 3D pour représenter des connexions et des flux sur une carte. Cette méthode de conception cartographique consiste à appliquer un angle au trait pour le transformer en courbe, lui donnant ainsi un aspect plus esthétique (Bahoken, 2016). Avec la 3D, cette incurvation du tracé peut être combinée à une élévation qui permet de donner à voir autrement les effets de flux (Dorling, 1991). En réponse à la multiplication de jeux de données sur les mobilités, l'utilisation des arcs 3D commence à se développer pour explorer les nombreuses matrices origines-destinations disponibles (figure 12). Sur le plan de l'interactivité, ce mode de représentation s'accompagne de fonctionnalités intéressantes, comme la sélection d'un sous-ensemble de données relatif à une zone de départ ou d'arrivée par le survol de la carte (brushing). Cette forme d'interaction est particulièrement bien adaptée à l'exploration de données de flux, car elle permet, par exemple, de visualiser au survol d'une zone, toutes les origines ou toutes les destinations associées. 
Figure 12. (a) Utilisation d'une station de vélos en libre-service à New York ; (b) Mobilités quotidiennes professionnelles en Ille-et-Vilaine ; (c) Migrations interurbaines aux États-Unis ; (d) Migrations pendulaires au Royaume-Uni
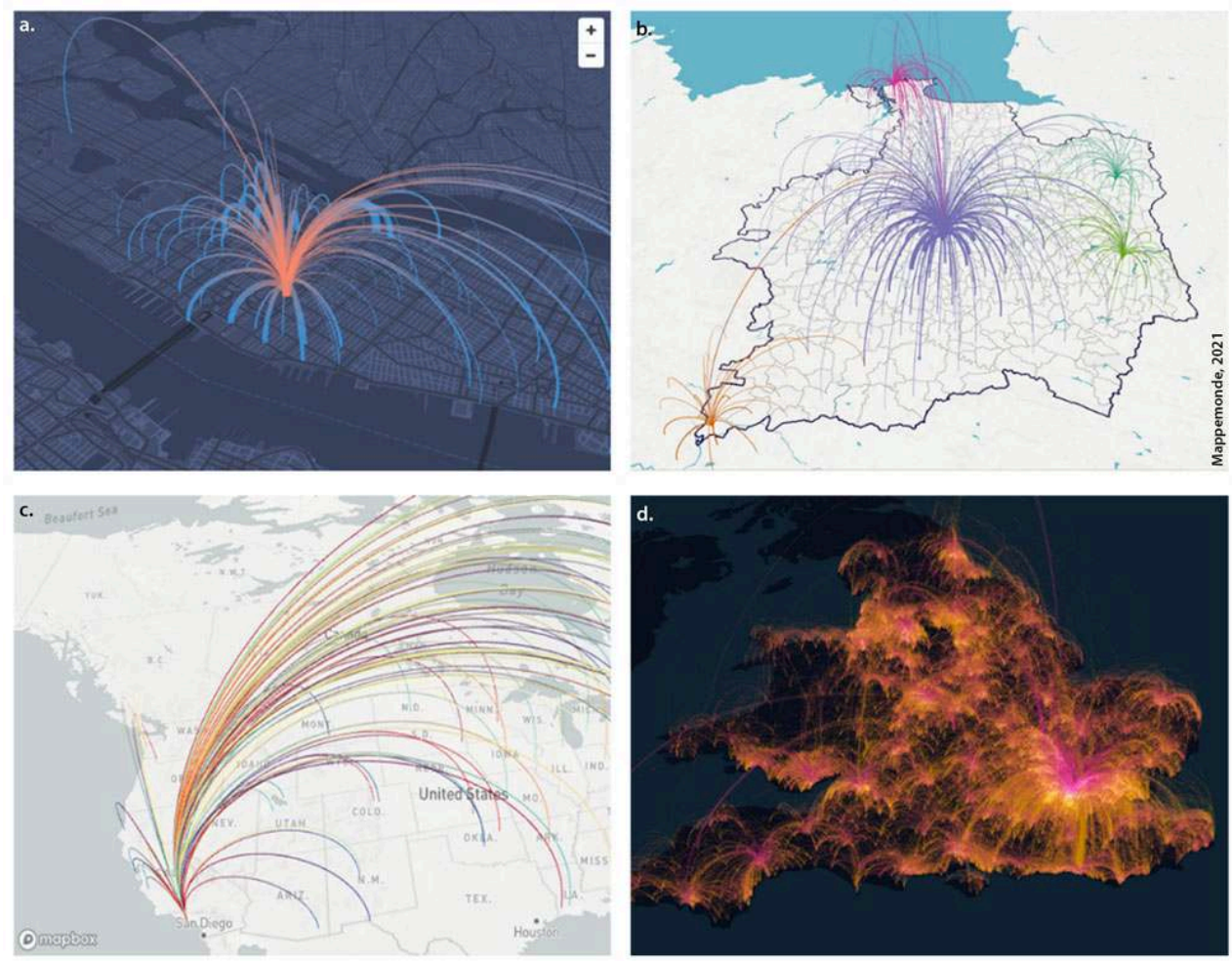

(a) Flourish : https://flourish.studio/2018/11/16/arc-map-webgl/ ; (b) Boris Mericskay : https:// bmericskay.github.io/portfolio/WebGL.html ; (c) DeckGL : https://deck.gl/\#/examples/core-layers/arclayer ; (d) KeplerGL : https://kepler.gl/demo/ukcommute

Avec le WebGL, il est désormais plus simple de proposer des cartes en ligne en 3D avec des rendus graphiques et des temps de chargement performants pour proposer aux usagers des géovisualisations plus interactives. Toutefois, cette approche présente des limites, notamment sur le plan de la lisibilité et de l'interprétation de l'intégralité des données représentées. Certains éléments de la carte peuvent être obstrués par des entités extrudées, limitant ainsi une lecture complète des données. Selon le nombre d'unités spatiales représentées, la carte peut également être plus ou moins expressive. Un trop grand nombre d'unités extrudées conduit en effet à produire une image très fragmentée et parfois difficile à interpréter (Cauvin et al., 2010). De plus, la capacité d'orientation au sein des cartes peut se voir affectée par un manque de points de repère univoques et conventionnels. L'interactivité propose une réponse à ce problème en permettant aux utilisateurs de se déplacer, de pivoter, d'incliner la vue et de changer d'échelle afin d'explorer et de naviguer dans ces nouveaux paysages de données. La troisième dimension offre en ce sens des formes renouvelées d'interaction avec les données spatiales, lesquelles peuvent contribuer à augmenter la perception et la compréhension du phénomène représenté (Christophe, 2017).

\section{Conclusion}

53 À la fois à l'origine d'améliorations au niveau des performances d'affichage, de la représentation comme de l'interaction, les avancées techniques exposées ici sont dans 
le même temps source de mauvaises pratiques (erreurs de sémiologie graphique, méconnaissance et mauvaise utilisation des méthodes, cartes peu lisibles, etc.). Et de manière générale, il en ressort que l'enjeu principal de la géovisualisation des données massives est davantage de proposer des environnements techniques et des modes de représentation adaptés aux volumes des données que de mettre à disposition des outils pour produire des représentations cartographiques efficientes.

L'une des principales innovations que connait la cartographie en ligne se situe au niveau des progrès autour des capacités de traitement et de rendu graphique à l'image du tuilage vectoriel et du WebGL. La combinaison de ces deux techniques permet à la fois d'optimiser la transmission et l'affichage de gros volumes de données spatiales et facilite dans le même temps le recours à des modes de représentation complexes à l'image de la 3D. De plus, ces techniques offrent également la possibilité d'agréger à la volée, sous des formes variées, des millions d'objets géographiques pour faciliter leur diffusion et leur lecture. Certaines opérations qui peuvent prendre plusieurs minutes voire plusieurs heures dans des SIG s'effectuent désormais en quelques secondes sur le géoweb.

Les avancées autour de l'interactivité entre l'individu, les données et les représentations constituent une autre innovation majeure. Les formes d'interactions sur le géoweb sont toujours plus variées tant au niveau des données (sélection, filtre) de la vue (déplacement, changement d'échelle, inclinaison, rotation) que des modes de représentation (choix du fond de carte, personnalisation des couleurs, changement de modes d'agrégation). Cette évolution conduit à positionner les cartes en ligne comme des instruments de navigation et d'exploration de masses d'informations spatiales où les lecteurs sont en capacité de construire leurs propres cartes.

En termes de modes de représentation, le développement des cartes choroplèthes en 3D, des carroyages, des arcs extrudés ou des clusters renvoient à des méthodes déjà connues et utilisées par les cartographes depuis de nombreuses années. Ces méthodes sont aujourd'hui transposées et remises au goût du jour au regard des innovations technologiques et des enjeux relatifs à la géovisualisation de données volumineuses. Il s'agit davantage d'une évolution des modes de représentation cartographique qui tient également pour beaucoup à la nouvelle génération de cartographes du Web pour qui la performance d'affichage (vitesse et fluidité) et l'aspect graphique sont importants. Les cartes en ligne sont, en effet, très axées autour de la Dataviz, où l'objectif est de proposer des visualisations esthétiques et séduisantes, souvent au détriment des « règles conventionnelles » de la cartographie thématique. Comme évoqué tout au long du texte, le recours à ces «nouveaux " modes de représentation induit surtout toute une série de questionnements relatifs à leur utilisabilité et à leur efficacité visuelle.

Mais le principal problème, au sein de ces environnements toujours plus techniques, réside dans le fait que les concepteurs de cartes en ligne ne disposent que d'assez peu de marge de manœuvre dans la configuration des géovisualisations (méthodes de discrétisation, choix de couleurs, paramétrage des agrégations ou des interpolations, fonctionnalités d'interaction...). Malgré une diversification des possibilités de mise en forme des données spatiales, on assiste à une certaine standardisation des modes de représentation, à l'image des cartes en cluster ou des cartes de chaleur qui sont toujours plus présentes sur le Web. Comme le rappellent Zanin et Ysebaert (2017), l'innovation en cartographie ne peut réellement émerger que si les moyens donnés au cartographe lui permettent de laisser sa créativité s'exprimer. Or, sur le géoweb la tendance est à la 
mise à disposition d'outils génériques déjà préconfigurés (solutions interfacées, bibliothèques logicielles, API), où les concepteurs de cartes sont pour beaucoup contraints par des cadres techniques et des modes de représentation de plus en plus normalisés. Une plus grande liberté de conception des cartes en ligne passe nécessairement par des compétences en programmation Web (HTML, CSS et JavaScript). Cette tendance à la technicisation de la cartographie en ligne questionne les cartographes et les géomaticiens qui doivent davantage s'investir dans cette mutation, tant d'un point de vue technique, méthodologique que thématique. Il est important que la cartographie sur le Web se renouvelle et évolue, mais il apparaît indispensable que la carte, comme moyen de communication, conserve ses spécificités et une certaine rigueur afin qu'elle ne devienne pas qu'un simple mode de visualisation de données en ligne parmi d'autres.

\section{BIBLIOGRAPHIE}

AMINI A., WAH T.Y., SAYBANI M.R., YAZDI S. (2011). “A study of density-grid based clustering algorithms on data streams". 8th International Conference on Fuzzy Systems and Knowledge Discovery, vol. 3, p. 1652-1656.

ANTONIOU V., MORLEY J., HAKLAY M.M. (2009). “Tiled vectors: A method for vector transmission over the web". In CARSWELL J.D., FOTHERINGHAM A.S., MCARDLE G., Web and Wireless Geographical Information Systems, p. 56-71.

BAHOKEN F. (2016). Contribution à la cartographie d'une matrice de flux. Thèse de doctorat de géographie, Paris : Université Paris Diderot, 408 p.

BERTIN J. (1967). Sémiologie graphique : Les diagrammes, les réseaux, les cartes. Paris : Mouton/ Gauthier-Villars, 428 p. ISBN 9782713224171

BEUKELAAR I.T.Y. (2018). Cartographic implications of Vector Tile technology. Mémoire de maîtrise en géomatique, Utrecht : Université d'Utrecht, $130 \mathrm{p}$.

BREUNIG M., BRADLEY P.E., JAHN M., KUPER P., MAZROO, N., RÖSCH N., JADIDI M., AL-DOORI M., STEFANAKIS E. (2020). “Geospatial Data Management Research: Progress and Future Directions”. ISPRS International Journal of Geo-Information, vol. 9, $\mathrm{n}^{\circ}$ 2, p. 95.

CAUVIN C., ESCOBAR F., SERRADJ A., ANTONI J.-P., KLEIN O. (2010). Cartography and the impact of the quantitative revolution. Vol. 2. Londres : Wiley-ISTE, 432 p. ISBN 1118586948

CRAMPTON, J.W., GRAHAM, M., POORTHUIS, A., SHELTON, T., STEPHENS, M., WILSON, M.W., ZOOK, M. (2013). "Beyond the geotag: situating 'big data' and leveraging the potential of the geoweb". Cartography and geographic information science, vol. 40, $\mathrm{n}^{\circ} 2$, p. 130-139.

CHRISTOPHE S. (2017). Personnalisation des représentations cartographiques en géovisualisation: couleurs et styles. Mémoire d'habilitation à diriger des recherches en Sciences et Technologies de l'Information géographique, Marne-la-Vallée : Université Paris-Est, 148 p.

DEBOER M. (2015). “Understanding the heat map”. Cartographic perspectives, nº 80, p. 39-43. 
DORLING D.F.L. (1991). The visualisation of spatial social structure. Thèse de doctorat, Newcastle : Université de Newcastle upon Tyne, 577 p.

DUBRAVA, T. (2017). Design of a multi-scale base map for a tiled web map service. Mémoire de maîtrise en cartographie, Enschede : Université de Twente, 93 p.

ELWOOD S. (2011). "Geographic information science: Visualization, visual methods, and the geoweb". Progress in Human Geography, vol. 35, n³ 3, p. 401-408.

ELWOOD S., LESZCZYNSKI A. (2013). "New spatial media, new knowledge politics". Transactions of the Institute of British Geographers, vol. 38, no 4, p. 544-559.

FARKAS G. (2017). “Applicability of open-source web mapping libraries for building massive Web GIS clients". Journal of Geographical Systems, vol. 19, n 3, p. 273-295.

FARKAS, G. (2019). “Hardware-Accelerating 2D Web Maps: A Case Study”. Cartographica: The International Journal for Geographic Information and Geovisualization, vol. 54, $\mathrm{n}^{\circ}$ 4, p. 245-260.

FEKETE J.D., PLAISANT C. (2002). “Interactive information visualization of a million items”. IEEE Symposium on Information Visualization - INFOVIS 2002. p. 117-124.

FRANKLIN R., LEWIS H. (1978). “3-D graphic display of discrete spatial data by prism maps”. ACM SIGGRAPH Computer Graphics, vol. 12, n 3, p. 70-75.

FÜRHOFF L. (2019). "Rethinking the usage and experience of clustering markers in web mapping". PeerJ Preprints : https://peerj.com/preprints/27858/

GAFFURI J. (2011). “Improving web mapping with generalization”. Cartographica: The International Journal for Geographic Information and Geovisualization, vol. 46, nº 2, p. 83-91.

GAFFURI, J. (2012). “Toward web mapping with vector data”. In XIAO N., KWAN M., GOODCHILD M.F., SHEKHAR S., GIScience 2012 : Geographic Information Science, p. 87-101.

HAYAT F. (2017), « Entre cartographie contributive et cartographie éditoriale ». Cartes et géomatique, $\mathrm{n}^{\circ} 233$, p. 9-17.

JENKS G.F. (1963). “Generalization in statistical mapping”. Annals of the Association of American Geographers, vol. 53, $\mathrm{n}^{\circ}$ 1, p. 15-26.

JÉGOU L. (2007). « La troisième dimension en cartographie statistique, des cartes en prismes imprimées aux modèles 3D interactifs ». Mappemonde, $\mathrm{n}^{\circ}$ 86, vol. 2. En ligne : http:// mappemonde-archive.mgm.fr/num14/articles/art07202.html

JEŽEK J., JEDLIČKA K., MILDORF T., KELLAR J., BERAN D. (2017). “Design and Evaluation of WebGL-Based Heat Map Visualization for Big Point Data”. In IVAN I., SINGLETON A., HORÁK J., INSPEKTOR T. The Rise of Big Spatial Data, p. 13-26.

JOLIVEAU T., NOUCHER M., ROCHE S. (2013). «La cartographie 2.0, vers une approche critique d'un nouveau régime cartographique ». L'Information géographique, vol. 77, nº 4, p. 29-46.

KEIM D., ANDRIENKO G., FEKETE J. D., GÖRG C., KOHLHAMMER J., MELANÇON G. (2008). “Visual analytics: Definition, process, and challenges”. In KERREN 1., STASKO J.T., FEKETE J.D., NORTH C., Information visualization, p. 154-175.

KIEFER A. (2015). Real time point cluster solutions in web mapping applications. Thèse de doctorat en géographie, université de Vienne, $115 \mathrm{p}$.

KORPI J., AHONEN-RAINIO P. (2013). "Cluster reduction methods for point symbols in map mashups". The Cartographic Journal, vol. 50, $\mathrm{n}^{\circ}$ 3, p. 257-265. 
KRÄMER M., GUTBELL, R. (2015). “A case study on 3D geospatial applications in the web using stateof-the-art WebGL frameworks". Proceedings of the 20th International Conference on 3D Web Technology, p. 189-197.

LAFRANCE F., DANIEL S., DRAGIĆEVIĆ S. (2019). “Multidimensional Web GIS Approach for Citizen Participation on Urban Evolution". ISPRS International Journal of Geo-Information, vol. 8, $\mathrm{n}^{\circ}$ 6, 253. LAMBERT N., ZANIN C. (2016). Manuel de cartographie : principes, méthodes, applications. Paris : Armand Colin, 224 p. ISBN $220061697 X$

LI L., HU W., ZHU H., LI Y., ZHANG H. (2017). “Tiled vector data model for the geographical features of symbolized maps". PloS ONE, vol. 12, n ${ }^{\circ} 5$.

LIENERT C., JENNY B., SCHNABEL O., HURNI L. (2012). "Current trends in vector-based Internet mapping: A technical review”. In Petterson M. (éd.), Online maps with APIs and WebServices, p. 23-36.

MEERT W. (2006) Clustering Maps. Mémoire de maîtrise en intelligence artificielle, Université Catholique de Louvain, $63 \mathrm{p}$.

MEIER S., HEIDMANN F., THOM A. (2014). "Heattile, a new method for heatmap implementations for mobile web-based cartographic applications”. In BRANDOVA T., KONECNY M., ZLATANOVA S. (éd.), Thematic Cartography for the Society, Springer, p. 33-44.

MEIER S. (2016). "The Marker Cluster: A Critical Analysis and a New Approach to a Common Webbased Cartographic Interface Pattern". International Journal of Agricultural and Environmental Information Systems, vol. 7, $\mathrm{n}^{\circ}$ 1, p. 28-43.

MERICSKAY B. (2016). « La cartographie à l'heure du géoweb : retour sur les nouveaux modes de représentation spatiale des données numériques ». Cartes et géomatique, ${ }^{\circ}$ 229-230, p. 37-50.

MUEHLENHAUS I. (2013). Web cartography: map design for interactive and mobile devices. Boca Raton : CRC Press, 262 p. ISBN 9781439876220

NETEK R., BRUS J., TOMECKA O. (2019). “Performance Testing on Marker Clustering and Heatmap Visualization Techniques: A Comparative Study on JavaScript Mapping Libraries". ISPRS International Journal of Geo-Information, vol. 8, $\mathrm{n}^{\circ}$ 8, p. 348.

NETEK R., MASOPUST J., PAVLICEK F., PECHANEC V. (2020). “Performance Testing on Vector vs. Raster Map Tiles. Comparative Study on Load Metrics". ISPRS International Journal of Geo-Information, vol. 9, $\mathrm{n}^{\circ} 2$, p. 101.

NGUYEN Q.D., DEVAUX A., BREDIF M., PAPARODITIS N. (2015). “3D heterogeneous interactive web mapping application". 2015 IEEE Virtual Reality (VR), p. 323-324.

PACHINGER F., SHEIKH Z., ZAJACZKOWSKI, P., ROGGE-SOLTI A., SCHONIG S., MENDLING J. (2016). “Comparison of Visualization Concepts of Map Layouts". 2016 IEEE 20th International Workshop on Enterprise Distributed Object Computing Workshop, p. 299-303.

POPELKA S. (2018). "Eye-tracking evaluation of 3D thematic maps". Proceedings of the 3rd Workshop on Eye Tracking and Visualization, p. 1-5.

RASE W.D. (2009). Visualization of polygon-based data as a continuous surface. Manuscrit.

REZAEI M., FRANTI P. (2018). "Real-Time Clustering of Large Geo-Referenced Data for Visualizing on Map". Advances in computer and electrical engineering, vol. 18, $\mathrm{n}^{\circ} 4, \mathrm{p} .63-74$.

SCHMIDT M., WEISER P. (2012). “Web mapping services: development and trends”. In PETERSON M. (éd.), Online maps with APIs and WebServices, Berlin, Heidelberg : Springer, p. 13-21. 
SHNEIDERMAN B. (1996). "The eyes have it: A task by data type taxonomy for information visualizations”. Proceedings 1996 IEEE symposium on visual languages, p. 336-343.

SHANG X. (2015). A Study on Efficient Vector Mapping With Vector Tiles Based on Cloud Server Architecture. Mémoire de maîtrise en génie géomatique, Calgary : Université de Calgary, 95 p. SINGLETON A., BRUNSDON C. (2014). "Escaping the pushpin paradigm in geographic information science:(re) presenting national crime data". Area, vol. 46, n 3, p. 294-304.

SLOCUM T., MC MASTER R., KESSLER F., HOWARD H. (2009). Thematic cartography and Geographic Visualization (3rd ed.). Upper Saddle River : Pearson, 576 p. ISBN 978-0132298346

STEWART J., KENNELLY P.J. (2010). "Illuminated choropleth maps". Annals of the Association of american Geographers, vol. 100, n 3, p. 513-534.

TARALDSVIK M. (2012). The future of web-based maps: can vector tiles and HTML5 solve the need for highperformance delivery of maps on the web? Mémoire de maitrise, Trondheim : Norwegian University of Science and Technology, $139 \mathrm{p}$.

TRAME J., KESSLER C. (2011). “Exploring the Lineage of Volunteered Geographic Information with Heat Maps". GeoViz 2011, Hambourg.

VEENENDAAL B., BROVELLI M. A., LI S. (2017). "Review of web mapping: Eras, trends and directions". ISPRS International Journal of Geo-Information, vol. 6, $\mathrm{n}^{\circ}$ 10, 317.

WARE C., PLUMLEE M. (2005). “3D geovisualization and the structure of visual space”. In DYKES J., MACEACHREN A., KRAAK J., Exploring Geovisualization. Amsterdam : Elsevier, p. 567-576.

ISBN 9780080531472

WHITE T.M. (2012). Evaluating the effectiveness of thematic mapping on virtual globes. Mémoire de maîtrise en géographie, Kansas City : University of Kansas. 179 p.

YAO X., LI G. (2018). “Big spatial vector data management: a review”. Big Earth Data, vol. 2, nº 1, p. $108-129$.

ZANIN C., YSEBAERT R. (2017). « Mise en place d'un modèle cartographique : Trouver le bon compromis entre homogénéisation et innovation cartographique ». Cartes et Géomatique, $\mathrm{n}^{\circ} 233$, p. 33-44.

ZOUHAR F., SENNER I. (2019). “Web-Based Visualization of Big Geospatial Vector Data”. Proceedings of the 22nd AGILE Conference on Geographic Information Science, p. 59-74.

\section{NOTES}

1. https://test.map.geo.admin.ch/

2. https://www.ordnancesurvey.co.uk/business-government/products/open-zoomstack

3. Etalab est une administration publique française qui fait office de Chief Data Officer de l'État et coordonne la conception et la mise en œuvre de sa stratégie dans le domaine de la donnée. https://openmaptiles.geo.data.gouv.fr/

4. https://geoservices.ign.fr/documentation/services_betas/vecteur-tuile.html

5. La généralisation cartographique s'appuie sur des algorithmes de simplification comme celui de Douglas-Peucker ou Visvalingam.

6. https://www.comeetie.fr/galerie/francepixels2019/\#5.7/47/2.3

7. https://map.onesoil.ai/2018\#2/44.35/-43.66

8. https://colouring.london/ 
9. https://cadastre.data.gouv.fr/map?style=ortho\#5/46.9/1.7

10. https://app.dvf.etalab.gouv.fr/

11. https://www.mapbox.com/boundaries\#coverageMap

12. https://www.mapbox.com/elections

13. http://maps.stamen.com/\#terrain/12/37.7706/-122.3782

14. https://www.mapzen.com/

15. https://snazzymaps.com/explore

16. https://www.mapbox.com/gallery/

17. https://openmaptiles.org/styles/

18. https://www.mapbox.com/mapbox-studio/

19. https://maps.jawg.io/\#8/48.863/2.359

20. https://developers.arcgis.com/vector-tile-style-editor/

21. https://mapstyle.withgoogle.com/

22. https://maputnik.github.io/

23. https://www.microsoft.com/en-us/p/map-style-sheet-editor/9nbhtcjt72ft? activetab=pivot:overviewtab

24. https://docs.mapbox.com/mapbox-gl-js/example/toggle-worldviews/

25. Les bibliothèques JavaScript de cartographie en ligne comme OpenLayers ou Leaflet offrent la possibilité de mobiliser des tuiles vectorielles. Idem pour les solutions de diffusion en ligne de données spatiales comme GeoServer qui permet de générer des flux de tuiles vectorielles. Enfin, l'Open Geospatial Consortium, dont l'objectif et de développer et de promouvoir des standards ouverts d'échange de données géographiques (comme le WMS), a récemment lancé un chantier autour de la mise en place d'un standard dédié aux tuiles vectorielles.

26. Dans le domaine des données spatiales, Meert (2006) identifie trois principales techniques de regroupement: (1) les méthodes basées sur les centroïdes telles que les algorithmes des kmoyennes; (2) les méthodes de regroupement hiérarchique ; (3) les méthodes basées sur les densités comme l'algorithme de partitionnement DBSCAN. La méthode la plus utilisée actuellement au sein des applications en ligne et des bibliothèques JavaScript est celle du regroupement hiérarchique, considérée comme la plus efficace (Fürhoff, 2019). Elle a notamment été popularisée par le développeur du plugin de regroupement MarkerCluster de Leaflet et reprise par la suite par les développeurs de Mapbox.

27. https://fr.wikipedia.org/wiki/JavaScript

28. https://fr.wikipedia.org/wiki/Acc\%C3\%A9l\%C3\%A9ration_mat\%C3\%A9rielle

29. https://cesium.com/platform/cesiumjs/

30. https://github.com/tangrams/tangram

31. https://docs.mapbox.com/mapbox-gl-js/example/

32. https://developers.arcgis.com/javascript/latest/sample-code/scene-webgl-support/

33. https://deck.gl/\#/

34. https://www.harp.gl/

35. http://www.itowns-project.org/

36. https://www.ogc.org/standards/3DTiles

37. Building Information Modeling.

38. https://pudding.cool/2018/10/city_3d/

39. https://pudding.cool/2018/12/3d-cities-story/?source=post_page-- 


\section{RÉSUMÉS}

Les avancées techniques autour de la visualisation de données volumineuses et l'affichage en 3D au sein de navigateurs Web viennent renouveler les pratiques de géovisualisation. Des modes basés sur l'agrégation à l'extrusion 3D en passant par les fonds de cartes personnalisés, le visage des cartes en ligne se transforme. Afin de bien comprendre cette forme de cartographie émergente et les enjeux sous-jacents, cet article questionne les logiques et les modes de représentation cartographique des données volumineuses qui prennent forme sur le Web au prisme des technologies émergentes et des usages associés.

Technical advances in big data and web-based 3D visualisation are reinventing geovisualisation practices. From modes based on spatial aggregation, 3D extrusion, to custom basemaps, the face of webmapping is changing. In order to fully understand this new form of mapping and the underlying issues, this article questions the logic and the modes of cartographic representation of big data that take form on the Web through the lens of emerging technologies and associated uses.

Las prácticas de geovisualización están mutando gracias a los avances tecnológicos de visualización de los macrodatos (big data), o por los levantamientos en 3D en los navegadores web. La cartografía en línea se está transformando al personalizar las bases cartográficas, los algoritmos de agregación y tratamiento masivo de la información, o por las posibilidades de representación tridimensional. En este artículo se reflexiona sobre las lógicas y los modos de representación espacial de los macrodatos que se visualizan con las nuevas tecnologías en la web. Pero también se especula sobre los nuevos usos y utilidades, así como los problemas subyacentes que emergen.

\section{INDEX}

Keywords : webmapping, big data, geovisualiation, vector tiles, WebGL, aggregation, 3D

Palabras claves : cartografía en línea, macrodatos (big data), geovisualización, teselas vectoriales, WebGl, agregación, 3D

Mots-clés : cartographie en ligne, données massives, géovisualisation, tuiles vectorielles, WebGL, agrégation, 3D

Thèmes : Comprendre l'innovation en cartographie

\section{AUTEUR}

\section{BORIS MERICSKAY}

Maître de conférences en géographie, Université Rennes 2, UMR Espaces et Sociétés (ESO-Rennes) 